\title{
NON-ARCHIMEDEAN AND TROPICAL THETA FUNCTIONS
}

\author{
TYLER FOSTER, JOSEPH RABINOFF, FARBOD SHOKRIEH, AND ALEJANDRO SOTO
}

\begin{abstract}
We define a tropicalization procedure for theta functions on abelian varieties over a non-Archimedean field. We show that the tropicalization of a non-Archimedean theta function is a tropical theta function, and that the tropicalization of a non-Archimedean Riemann theta function is a tropical Riemann theta function, up to scaling and an additive constant. We apply these results to the construction of rational functions with prescribed behavior on the skeleton of a principally polarized abelian variety. We work with the Raynaud-Bosch-Lütkebohmert theory of non-Archimedean theta functions for abelian varieties with semi-abelian reduction.
\end{abstract}

\section{INTRODUCTION}

Let $K$ be a nontrivially valued, complete, algebraically closed, non-Archimedean field. Let $A$ be an abelian variety over $K$. Suppose for the moment that $A$ is principally polarized with totally degenerate reduction. In this case, the non-Archimedean uniformization theory of $A$ essentially amounts to a finitely generated, free abelian group $M$, and a symmetric pairing $t(\cdot, \cdot): M \times M \rightarrow K^{\times}$, such that $-\log |t|$ is positive-definite. This pairing defines a homomorphism from $M$ to the torus $\mathbf{T}=\operatorname{Spec} K[M]$ by the rule $\iota: u \mapsto x_{u}$, where $x_{u}\left(\chi^{u^{\prime}}\right)=t\left(u, u^{\prime}\right)$. (Here $\chi^{u^{\prime}}: \mathbf{T} \rightarrow \mathbf{G}_{m}$ is the character corresponding to $u^{\prime} \in M$ ). Then the Berkovich analytification $A^{\text {an }}$ is canonically isomorphic to the quotient $\mathbf{T}^{\text {an }} / \iota(M)$. In this setting, a (non-Archimedean) Riemann theta function for $A$ is the analytic function $f: \mathbf{T}^{\text {an }} \rightarrow \mathbf{A}^{1, \text { an }}$ given by the convergent power series

$$
f=\sum_{u \in M} \sqrt{t(u, u)} \chi^{u}
$$

with respect to some choice of $\sqrt{t}$.

Berkovich [Ber90] showed that the analytification $A^{\text {an }}$ contains a canonical subset $\Sigma$, called a skeleton, onto which it deformation retracts. This skeleton is a tropical abelian variety (Definition 2.6, Proposition 4.7): it is a real torus with an integral structure that admits a "polarization". A principal polarization of $A$ induces a principal polarization of $\Sigma$, in which case $\Sigma$ amounts to the data of a finitely generated, free abelian group $M$ equipped with a positive-definite, symmetric bilinear pairing $[\cdot, \cdot]: M \times M \rightarrow \mathbf{R}$. This pairing defines an injective homomorphism $\iota: M \rightarrow N_{\mathbf{R}}=\operatorname{Hom}(M, \mathbf{R})$ by $\iota(u)=\left(u^{\prime} \mapsto\right.$ $\left.\left[u, u^{\prime}\right]\right)$; the real torus in question is $\Sigma=N_{\mathrm{R}} / \iota(M)$. The tropical Riemann theta function for $\Sigma$ is the piecewise linear function $\varphi: N_{\mathrm{R}} \rightarrow \mathbf{R}$ defined by the formula

$$
\varphi(v)=\min _{u \in M}\left\{\frac{1}{2}[u, u]+\langle u, v\rangle\right\},
$$

where $\langle\cdot, \cdot\rangle: M \times N_{\mathbf{R}} \rightarrow \mathbf{R}$ is the evaluation pairing. 
In this paper, we make the obvious analogy between $(*)$ and $(* *)$ into a precise relationship. We do not assume $A$ is principally polarized, and more importantly, we allow $A$ to have arbitrary mixed reduction type. It is still true that $A^{\text {an }}$ admits a canonical skeleton $\Sigma=N_{\mathrm{R}} / M^{\prime}$, which is a tropical abelian variety, but the theory of non-Archimedean theta functions in this case is very much more technical. We define a tropicalization procedure $f \mapsto f_{\text {trop }}$, which takes a theta function $f$ on $A^{\text {an }}$ and produces a piecewise-linear function $f_{\text {trop }}: N_{\mathbf{R}} \rightarrow \mathbf{R}$. We prove the following results:

Theorem A. The tropicalization of a non-Archimedean theta function is a tropical theta function.

See Definition 2.8 for the definition of a general tropical theta function, and see Theorem 4.10 for a precise statement of Theorem A.

Theorem B. If $f$ is the Riemann theta function associated to a principal polarization of A, then $f_{\text {trop }}$ is the tropical Riemann theta function associated to the induced principal polarization of $\Sigma$, up to translation and an additive constant.

See Theorem 4.13 for a precise statement.

The main interest in Theorems $\mathrm{A}$ and $\mathrm{B}$ is to construct rational functions on $A$ with a prescribed behavior on $\Sigma$. Given a nonzero rational function $f$ on $A$, one can simply restrict $-\log |f|$ to $\Sigma$, to obtain a piecewise linear function $f_{\text {trop }}: \Sigma \rightarrow \mathbf{R}$. Let $\left(f_{1}, \ldots, f_{n}\right)$ be a tuple of rational functions, and let $f: A \rightarrow \mathbf{G}_{m}^{n}$ be the rational map $\left(f_{1}, \ldots, f_{n}\right)$. Composing with the tropicalization map trop: $\mathbf{G}_{m}^{n \text {,an }} \rightarrow \mathbf{R}^{n}$, defined on points by $\left(x_{1}, \ldots, x_{n}\right) \mapsto$ $-\left(\log \left|x_{1}\right|, \ldots, \log \left|x_{n}\right|\right)$, gives a partially-defined function $A^{\text {an }} \rightarrow \mathbf{R}^{n}$. The restriction of this function to $\Sigma$ is the function $f_{\text {trop }}: \Sigma \rightarrow \mathbf{R}^{n}$ given by $f_{\text {trop }}(x)=\left(f_{1, \text { trop }}(x), \ldots, f_{n \text {,trop }}(x)\right)$. Careful construction of $\left(f_{1}, \ldots, f_{n}\right)$ yields a map $f_{\text {trop }}$ with nice properties (for example, unimodularity); this is the subject of future work of the authors.

In order to allow such constructions, we prove the following result.

Theorem C. Let $\varphi_{1}, \varphi_{2}$ be tropical theta functions for $\Sigma$ with the same automorphy factor. Suppose that $\varphi_{1}$ and $\varphi_{2}$ are tropicalizations of non-Archimedean theta functions. Then $\varphi_{1}-\varphi_{2}: \Sigma \rightarrow \mathbf{R}$ is the tropicalization of a nonzero rational function on $A$.

See Theorem 5.2 for a precise statement. In $\S 5$ we give concrete examples of constructions of rational functions on $A$ using Theorem C.

1.1. Notation. The following notations are used throughout the paper. We let $K$ denote an algebraically closed field which is complete with respect to a nontrivial, nonArchimedean absolute value $|\cdot|$. Let val $=-\log |\cdot|$ denote a corresponding valuation, $R$ the ring of integers of $K$, and $k$ its (algebraically closed) residue field.

We will generally use $M$ for a finitely-generated, free abelian group; we denote its dual by $N=\operatorname{Hom}(M, \mathbf{Z})$, and we set $N_{\mathbf{R}}=\operatorname{Hom}(M, \mathbf{R})=N \otimes_{\mathrm{Z}} \mathbf{R}$. The evaluation pairing is denoted $\langle\cdot, \cdot\rangle: M \times N_{\mathbf{R}} \rightarrow \mathbf{R}$. The monoid ring on $M \cong \mathbf{Z}^{n}$ is written $K[M] \cong$ $K\left[T_{1}^{ \pm 1}, \ldots, T_{n}^{ \pm 1}\right]$, and the character on $\operatorname{Spec}(K[M])$ corresponding to $u \in M$ is written $\chi^{u} \in K[M]$.

If $X$ is a finitely-type $K$-scheme, we denote by $X^{\text {an }}$ its analytification in the sense of Berkovich [Ber90]. For $x \in X^{\text {an }}$ we let $\mathscr{H}(x)$ denote the completed residue field at $x$. This is a complete valued field extension of $K$ with valuation ring $\mathscr{H}(x)^{\circ}$. 
Let $L$ be a line bundle on a $K$-scheme $X$ and let $x \in X(K)$ be a $K$-point. The $x$-fiber of $L$ is denoted $L_{x}$, and the constant line bundle on $L_{x}$ is $L(x):=L_{x} \times X$.

The special and generic fibers of an $R$-scheme $\mathfrak{X}$ are denoted $\mathfrak{X}_{s}$ and $\mathfrak{X}_{\eta}$, respectively.

Let $A$ be an abelian variety. The dual of $A$ is denoted $A^{\prime}$, and if $\varphi: A \rightarrow B$ is a homomorphism of abelian varieties then its dual is denoted $\varphi^{\prime}: B^{\prime} \rightarrow A^{\prime}$. For $x \in A(K)$ we let $T_{x}: A \rightarrow A$ be translation by $x$. A line bundle $L$ on $A$ determines a symmetric homomorphism $\varphi_{L}: A \rightarrow A^{\prime}$ defined on points by $x \mapsto T_{x}^{*} L \otimes L^{-1}$.

Acknowledgments. This project was completed as a part of the Mathematics Research Communities workshop held by the American Mathematical Society in the summer of 2013. The authors would like to thank the AMS for their support, and Matthew Baker and Sam Payne for organizing the workshop and for useful discussions. The third author would like to thank Alberto Bellardini for many helpful conversations. The authors gratefully thank the anonymous referee for carefully reading the paper and for providing some very helpful comments.

Foster was supported by NSF RTG grant DMS-0943832 and by Le Laboratoire d'Excellence CARMIN. Rabinoff was supported by NSF DMS-1601842. Soto would like to thank to the KU Leuven and to the Goethe Universität Frankfurt am Main for the great working conditions.

\section{TROPICAL ABELIAN VARIETIES AND TROPICAL THETA FUNCTIONS}

In this section we fix our notions regarding real tori with integral structure, tropical abelian varieties, and tropical theta functions. We define the tropical Riemann theta function associated to a principally polarized tropical abelian variety.

2.1. Real tori with integral structure. We begin by defining real tori with integral structure, a weaker notion than a tropical abelian variety which is sufficient to define piecewise linear functions.

Definition 2.2. Let $N$ be a finitely generated, free abelian group, let $N_{\mathbf{R}}=N \otimes_{\mathrm{Z}} \mathbf{R}$, and let $\Lambda \subset N_{\mathrm{R}}$ be a (full rank) lattice. The quotient $\Sigma=N_{\mathrm{R}} / \Lambda$ is called a real torus with integral structure.

The "integral structure" in $\Sigma$ is the choice of lattice $N \subset N_{\mathrm{R}}$, which need not coincide with the quotient lattice $\Lambda$.

Definition 2.3. Let $N, N^{\prime}$ be finitely generated, free abelian groups and let $N_{\mathrm{R}}=N \otimes_{\mathrm{Z}} \mathbf{R}$ and $N_{\mathrm{R}}^{\prime}=N^{\prime} \otimes_{\mathrm{Z}} \mathbf{R}$. Let $\Lambda \subset N_{\mathrm{R}}$ be a lattice and let $\Sigma=N_{\mathrm{R}} / \Lambda$, with quotient homomorphism $\pi: N_{\mathrm{R}} \rightarrow \Sigma$.

(1) A homomorphism $\varphi: N_{\mathbf{R}} \rightarrow N_{\mathrm{R}}^{\prime}$ is integral affine provided that $\varphi=\psi_{\mathbf{R}}+v$, where $\psi: N \rightarrow N^{\prime}$ is a homomorphism, $\psi_{\mathrm{R}}$ is the extension of scalars of $\psi$, and $v \in N_{\mathrm{R}}^{\prime}$.

(2) A continuous function $\varphi: N_{\mathrm{R}} \rightarrow N_{\mathrm{R}}^{\prime}$ is piecewise integral affine provided that $N_{\mathrm{R}}$ can be covered by full-dimensional polyhedra $\Delta$ such that $\left.\varphi\right|_{\Delta}$ is integral affine.

(3) A continuous function $\bar{\varphi}: \Sigma \rightarrow N_{\mathrm{R}}^{\prime}$ is piecewise integral affine provided that the composition $\varphi=\bar{\varphi} \circ \pi$ is piecewise integral affine. 
We say that a function $\varphi$ from $N_{\mathbf{R}}$ or $\Sigma$ to $\mathbf{R}^{r}$ has one of the above properties if it has that property with respect to the integral structure $N^{\prime}=\mathbf{Z}^{r} \subset \mathbf{R}^{r}$.

Some of the functions $\varphi: \Sigma \rightarrow N_{\mathrm{R}}^{\prime}$ arising in the sequel will be symmetric with respect to negation, so we make the following definitions.

Definition 2.4. With the notation in Definition 2.3, let $\varphi: \Sigma \rightarrow N_{\mathrm{R}}^{\prime}$ be a piecewise integral affine function. We say that $\varphi$ is Kummer provided that $\varphi(x)=\varphi(-x)$ for all $x \in \Sigma$.

In other words, a Kummer map $\varphi: \Sigma \rightarrow N_{\mathrm{R}}^{\prime}$ factors through the quotient $\Sigma \rightarrow \Sigma /(-1)$ by the negation action.

2.5. Tropical abelian varieties. Now we discuss tropical abelian varieties. These are real tori with integral structure which admit a polarization, in the sense defined below. The skeleton of an abelian variety is naturally a tropical abelian variety: see Proposition 4.7 below. Tropical abelian varieties and tropical theta functions have been implicit in the literature for years; see for instance Mumford [Mum72, Proposition 6.7] and Faltings-Chai [FC90, Definition 1.5, p.197]. The name "tropical abelian variety" seems to have been used first in [MZ08].

Definition 2.6. We fix the following data:

(a) Finitely generated free abelian groups $M, M^{\prime}$ of the same rank.

(b) A nondegenerate pairing $[\cdot, \cdot]: M^{\prime} \times M \rightarrow \mathbf{R}$.

The pairing defines $M^{\prime}$ (resp. $\left.M\right)$ as a lattice in $N_{\mathbf{R}}=\operatorname{Hom}(M, \mathbf{R})\left(\operatorname{resp} \cdot N_{\mathbf{R}}^{\prime}=\operatorname{Hom}\left(M^{\prime}, \mathbf{R}\right)\right)$. Suppose that there exists $\lambda: M^{\prime} \rightarrow M$ such that $[\cdot, \lambda(\cdot)]: M^{\prime} \times M^{\prime} \rightarrow \mathrm{R}$ is symmetric and positive-definite. Then the map $\varphi: N_{\mathrm{R}} \rightarrow N_{\mathrm{R}}^{\prime}$ dual to $\lambda$ takes $M^{\prime}$ into $M$ via $\lambda$. Under these conditions:

(1) We say that $\Sigma=N_{\mathrm{R}} / M^{\prime}$ is a tropical abelian variety.

(2) The dual of $\Sigma$ is the tropical abelian variety $\Sigma^{\prime}=N_{\mathrm{R}}^{\prime} / M$.

(3) The induced homomorphism $\bar{\varphi}: \Sigma \rightarrow \Sigma^{\prime}$ is called a polarization.

(4) We say that $\varphi$ is a principal polarization if $\lambda$ is an isomorphism.

The existence of $\lambda$ here plays the role of Riemann's period relations, which guarantee that a complex torus is in fact an algebraic variety. We leave it as an exercise to show that $\Sigma^{\prime}$ is in fact a tropical abelian variety. Since $N=\operatorname{Hom}(M, \mathbf{Z})$ is a lattice in $N_{\mathbf{R}}$, a tropical abelian variety is a real torus with integral structure.

2.7. Tropical theta functions. Finally we are able to define tropical theta functions. Recall that classically, a theta function is a holomorphic function on a complex vector space $V$ which is quasi-periodic with respect to a full dimensional lattice in $V$. The same is true in the non-archimedean setting. In the tropical side, a tropical theta function will be defined following the classical counterparts, i.e. as a piecewise linear function on a real vector space which is quasi-periodic with respect to a lattice. This quasi-periodicity condition should be thought as the tropical side of the non-archimedean one. This will made precise in $\S 4$.

Definition 2.8. Let $\Sigma=N_{\mathrm{R}} / M^{\prime}$ be a tropical abelian variety, as in Definition 2.6, and let $\lambda: M^{\prime} \rightarrow M$ be a homomorphism definining a polarization. Let $c: M^{\prime} \rightarrow \mathrm{R}$ be a function 
satisfying $c\left(u_{1}^{\prime}+u_{2}^{\prime}\right)-c\left(u_{1}^{\prime}\right)-c\left(u_{2}^{\prime}\right)=\left[u_{1}^{\prime}, \lambda\left(u_{2}^{\prime}\right)\right]$ for all $u_{1}^{\prime}, u_{2}^{\prime} \in M^{\prime}$. A tropical theta function with respect to $(\lambda, c)$ is a piecewise integral affine function $\varphi: N_{\mathbf{R}} \rightarrow \mathbf{R}$ satisfying the transformation law

$$
\varphi(v)=\varphi\left(v+u^{\prime}\right)+c\left(u^{\prime}\right)+\left\langle\lambda\left(u^{\prime}\right), v\right\rangle
$$

for all $u^{\prime} \in M^{\prime}$ and $v \in N_{\mathrm{R}}$.

Let $c$ be a function as in Definition 2.8. Then $u^{\prime} \mapsto c\left(u^{\prime}\right)-\frac{1}{2}\left[u^{\prime}, \lambda\left(u^{\prime}\right)\right]$ is a homomorphism $M^{\prime} \rightarrow \mathbf{R}$. In other words, $c\left(u^{\prime}\right)$ differs from $\frac{1}{2}\left[u^{\prime}, \lambda\left(u^{\prime}\right)\right]$ by a homomorphism.

Definition 2.9. With the notation in Definition 2.8, suppose now that $\lambda$ is an isomorphism. The tropical Riemann theta function associated to $\lambda$ is

$$
\varphi(v):=\min _{u^{\prime} \in M^{\prime}}\left\{\frac{1}{2}\left[u^{\prime}, \lambda\left(u^{\prime}\right)\right]+\left\langle\lambda\left(u^{\prime}\right), v\right\rangle\right\} .
$$

The tropical Riemann theta function $\varphi$ is in fact a tropical theta function with respect to $(\lambda, c)$ for $c\left(u^{\prime}\right)=\frac{1}{2}\left[u^{\prime}, \lambda\left(u^{\prime}\right)\right]$ : however, it is not obvious that $\varphi$ is piecewise integral affine or that it satisfies the stipulated transformation law. This will follow from Theorem 4.10, but this is not a reasonable proof. See [MZ08] for a full discussion of the tropical Riemann theta function.

\section{NON-ARCHIMEDEAN UNIFORMIZATION AND THETA FUNCTIONS}

Recall that $K$ is a complete and algebraically closed non-Archimedean valued field with valuation ring $R$. Let $A$ be an abelian variety over $K$. A theta function is almost the same as a global section of a line bundle $L$ on $A$. The reason for the name is that theta functions are constructed analytically on the universal cover of $A^{\text {an }}$, as in the classical situation over $\mathbf{C}$, where such functions are generally called $\theta$. This theory of non-Archimedean uniformizations and theta functions was worked out by Bosch and Lütkebohmert. It is a beautiful theory, but when $A$ has semi-abelian reduction it is quite technical. In this section we recall the results in [BL91] that we will use, translated from Bosch-Lütkebohmert's language of rigid and formal geometry into the language of Berkovich analytic spaces. We also prove a fact about Fourier coefficients of non-Archimedean theta functions in Proposition 3.25, which will be important in $\S 4$.

We discuss the totally degenerate case, which is much simpler, as a running example. The reader may want to understand this case first. See also Faltings-Chai [FC90], as well as Fresnel-van der Put [FvdP04] for a detailed discussion in the totally degenerate case.

If $L \rightarrow A$ is a line bundle then $L^{\text {an }} \rightarrow A^{\text {an }}$ is also a line bundle, and it follows from analytic GAGA that $H^{0}(A, L)=H^{0}\left(A^{\text {an }}, L^{\text {an }}\right)$. Moreover, any analytic line bundle on a proper variety is automatically algebraic. Hence we will sometimes neglect to distinguish between algebraic and analytic line bundles on abelian varieties.

3.1. Metrized line bundles. Let $\pi: L \rightarrow X$ be a line bundle on an analytic space. A metric on $L$ is a function $\|\cdot\|: L \rightarrow \mathbf{R}_{\geq 0}$ which is compatible with the $\mathbf{G}_{m}^{\text {an }}$-action and is non-constant on fibers: that is, given $x \in X$ and an isomorphism $\pi^{-1}(x) \cong \mathbf{A}_{\mathscr{H}(x)}^{1 \text {,an }}=$ $\operatorname{Spec}(\mathscr{H}(x)[T])^{\text {an }}$, for $y \in \pi^{-1}(x)$ we have $\|y\|=c|T(y)|$ for some $c \in\left|\mathscr{H}(x)^{\times}\right|$. We 
require the metric to be continuous in the sense that for $U \subset X$ open and a section $s:\left.U \rightarrow L\right|_{U}$, the function $x \mapsto\|s(x)\|: U \rightarrow \mathbf{R}_{\geq 0}$ is continuous.

Given a metrized line bundle $(L,\|\cdot\|)$ on $X$ and a morphism $\varphi: Y \rightarrow X$, there is an obvious metric on $\varphi^{*} L$. Given two metrized line bundles $\left(L_{1},\|\cdot\|_{1}\right)$ and $\left(L_{2},\|\cdot\|_{2}\right)$, there is a metric $\|\cdot\|$ on $L \otimes L^{\prime}$ characterized by $\left\|s_{1} \otimes s_{2}(x)\right\|=\left\|s_{1}(x)\right\|_{1}\left\|s_{2}(x)\right\|_{2}$.

An integral model induces a metric in the following way. Let $\mathfrak{L}$ be a line bundle on a flat, proper $R$-scheme $\mathfrak{X}$. Let $L$ be the generic fiber of $\mathfrak{L}$, i.e., the restriction of $\mathfrak{L}$ to $X:=\mathfrak{X} \times_{R} \operatorname{Spec}(K)$. In this situation we say that $\mathfrak{L}$ is an integral model of $L$ on $\mathfrak{X}$. The model metric $\|\cdot\|_{\mathfrak{L}}: L^{\text {an }} \rightarrow \mathbf{R}_{\geq 0}$ on $L^{\text {an }}$ associated to the model $\mathfrak{L}$ is defined as follows. Let $y \in L^{\text {an }}$ with image $x \in X^{\text {an }}$. By the valuative criterion of properness, $x$ extends to a unique $\mathscr{H}(x)^{\circ}$-point $x: \operatorname{Spec} \mathscr{H}(x)^{\circ} \rightarrow \mathfrak{X}$. Choosing any trivialization of $\mathfrak{L}$ in a neighborhood of the reduction of $x$ gives an isomorphism $T: x^{*} \mathfrak{L} \stackrel{\sim}{\longrightarrow} \mathbf{A}_{\mathscr{H}(x)^{\circ}}^{1}$. We set $\|y\|_{\mathfrak{L}}=|T(y)|$. This is well-defined because any other trivialization $T^{\prime}$ of $x^{*} \mathfrak{L}$ differs by a unit in $\mathscr{H}(y)^{\circ}$. See also [Gub10, §3].

Suppose now that $B$ is an abelian variety over $K$ with good reduction. Up to isomorphism, there is a unique abelian $R$-scheme $\mathfrak{B}$ whose generic fiber is identified with $B$. Let $L$ be a rigidified line bundle on $B$ (a line bundle with a trivialization of its identity fiber). It follows from [BL91, Lemma 6.1] and from the formal GAGA principle [FK17, §I.10] that $L$ has a canonical integral model $\mathfrak{L}$ on $\mathfrak{B}$, so there is a canonical model metric on $L^{\text {an }}$, which we denote by $\|\cdot\|_{L}$.

3.2. Raynaud-Bosch-Lütkebohmert uniformization. Let $A$ be an abelian variety over $K$. Let $p: E^{\text {an }} \rightarrow A^{\text {an }}$ be the universal cover (in the sense of topology), and choose a base point $0 \in E^{\text {an }}$ over the identity. Then $E^{\text {an }}$ has the unique structure of an analytic group with identity 0 , and $p$ is a homomorphism. In fact $E^{\text {an }}$ is the analytification of a group scheme $E$, although the homomorphism $p$ is not algebraic. The uniformization theory of Raynaud and Bosch-Lütkebohmert says that there are two exact sequences

$$
\begin{aligned}
& 0 \longrightarrow \mathrm{T} \longrightarrow E \stackrel{q}{\longrightarrow} B \longrightarrow 0 \\
& 0 \longrightarrow M^{\prime} \longrightarrow E^{\text {an }} \stackrel{p}{\longrightarrow} A^{\text {an }} \longrightarrow 0
\end{aligned}
$$

where $\mathbf{T}=\operatorname{Spec}(K[M])$ is a split $K$-torus, $B$ is an abelian variety with good reduction, and $M^{\prime}$ is a lattice in $E^{\text {an }}$ (see below). Moreover, all of these data are uniquely determined by $A$, up to isomorphism. The sequence (1) is called a Raynaud extension. Both sequences are often written in a so-called Raynaud cross

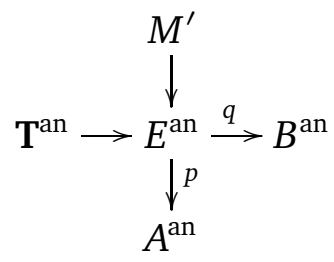


Given $u \in M$, we construct the pushout diagram

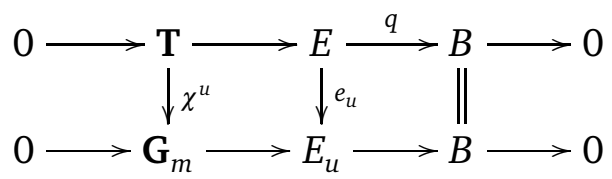

A rigidified translation-invariant line bundle on an abelian variety $B$ amounts to an extension of $B$ by $\mathbf{G}_{m}$ : given such an extension $L^{\prime}$, the $\mathbf{G}_{m}$-action makes $L^{\prime}$ into a $\mathbf{G}_{m}$-torsor over $B$, which is then identified with the complement of the zero section of a line bundle $L$. This line bundle is translation-invariant: given $x \in B$, choose a lift $y \in L^{\prime}$; then multiplication by $y$ in $L^{\prime}$ yields an isomorphism $L \stackrel{\sim}{\longrightarrow} x^{*} L$. Thus $E_{u}$ is the complement of the zero locus of such a rigidified translation-invariant line bundle on $B$; we also denote this line bundle (with zero section included) by $E_{u}$ by abuse of notation. Note that we may regard $e_{u}$ as a trivialization of the pullback $q^{*} E_{u}$.

Now we explain the lattice condition. Define the tropicalization map trop: $\mathbf{T}^{\mathrm{an}} \rightarrow N_{\mathbf{R}}$ by the rule

$$
\langle u, \operatorname{trop}(x)\rangle:=-\log \left|\chi^{u}(x)\right|, \quad u \in M .
$$

This extends in a canonical way to a homomorphism trop: $E^{\text {an }} \longrightarrow N_{\mathbf{R}}$ by setting

$$
\langle u, \operatorname{trop}(x)\rangle=-\log \left\|e_{u}(x)\right\|_{E_{u}},
$$

where $\|\cdot\|_{E_{u}}$ is the model metric defined in (3.1) using the fact that $B$ has good reduction. To say that $M^{\prime}$ is a lattice means that trop maps $M^{\prime}$ isomorphically onto an ordinary lattice (of full rank) in the Euclidean space $N_{\mathrm{R}}$.

Remark 3.3. The identity fiber of trop: $E^{\text {an }} \rightarrow N_{\mathbf{R}}$ is a subgroup $A_{0}$ of $E^{\text {an }}$, and we have a short exact sequence

$$
0 \longrightarrow \mathbf{T}_{0} \longrightarrow A_{0} \stackrel{q_{0}}{\longrightarrow} B^{\text {an }} \longrightarrow 0
$$

with $\mathbf{T}_{0}=\operatorname{trop}^{-1}(0)$, the affinoid torus. We call the sequence (3.3.1) a formal Raynaud extension because it arises as the generic fiber of a short exact sequence of formal group schemes over $R$. This sequence splits locally in the formal analytic topology on $B^{\text {an }}$ : that is, there exists a cover of $B^{\text {an }}$ by open sets $V$ which are the generic fiber of a formal affine, such that $q_{0}^{-1}(V) \cong \mathbf{T}_{0} \times V$. Extending the splitting to $q^{-1}(V) \cong \mathbf{T} \times V$, the map trop: $q^{-1}(V) \rightarrow N_{\mathbf{R}}$ is the composition of the first projection $\mathbf{T} \times V \rightarrow \mathbf{T}$ with trop: $\mathbf{T} \rightarrow N_{\mathbf{R}}$. See [BL91, §1] and [Gub10, (4.2)] for details.

The two extremal cases for the uniformization theory of $A$ are when $A$ has good reduction, in which case $\mathbf{T}$ and $M^{\prime}$ are trivial and $A=B$, and when $A$ has totally degenerate (i.e., toric) reduction, in which case $B=0$ and $E=\mathbf{T}$. It is precisely this later situation which is analogous to the uniformization of abelian varieties defined over the complex numbers, as we have $A^{\text {an }} \cong \mathrm{T}^{\text {an }} / M^{\prime}$.

3.4. Duality theory. Let $A$ be an abelian variety with dual $A^{\prime}$. Much of [BL91] is concerned with relating the uniformizations of $A$ and $A^{\prime}$. The end result is that we have the 
following Raynaud crosses:
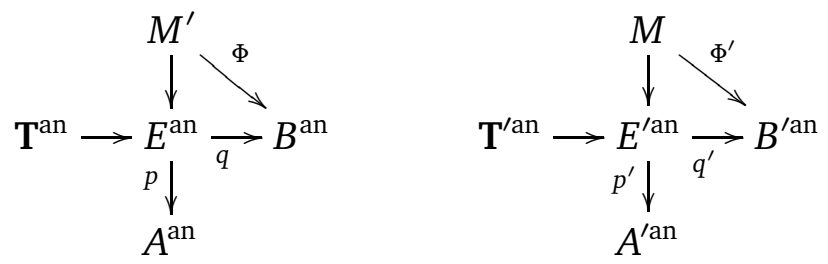

Here $\mathbf{T}=\operatorname{Spec}(K[M])$ and $\mathbf{T}^{\prime}=\operatorname{Spec}\left(K\left[M^{\prime}\right]\right)$, and $B$ is the dual of $B^{\prime}$. The map $\Phi^{\prime}$ has the property that $\Phi^{\prime}(u)=E_{u}$ (regarded as a $K$-point of $B^{\prime}$ ), and likewise for $\Phi$. Hence for $\left(u^{\prime}, u\right) \in M^{\prime} \times M$ we have $e_{u}\left(u^{\prime}\right), e_{u^{\prime}}(u) \in P_{\Phi\left(u^{\prime}\right) \Phi^{\prime}(u)}$, the fiber over $\left(\Phi\left(u^{\prime}\right), \Phi^{\prime}(u)\right)$ of the Poincaré bundle $P \rightarrow B \times B^{\prime}$. It turns out that $e_{u}\left(u^{\prime}\right)=e_{u^{\prime}}(u)$, and the map

$$
\left(u^{\prime}, u\right) \mapsto t\left(u^{\prime}, u\right):=e_{u}\left(u^{\prime}\right)=e_{u^{\prime}}(u) \quad t: M^{\prime} \times M \longrightarrow P
$$

is a trivialization of $\left(\Phi \times \Phi^{\prime}\right)^{*} P$, regarded as a biextension of $M^{\prime} \times M$. The valuation

$$
[\cdot, \cdot]:=-\log \|t(\cdot, \cdot)\|_{P}: M^{\prime} \times M \longrightarrow \mathbf{R}
$$

is bilinear and nondegenerate by [BL91, Proposition 3.4].

We use the notation introduced above for the rest of this section.

Remark 3.5. In [BL91] the roles of $M$ and $M^{\prime}$ are reversed. We chose to follow the opposite convention since in their situation, the tropicalization map on $E^{\text {an }}$ would take values in $N_{\mathrm{R}}^{\prime}$, which is nonstandard from the tropical point of view.

Example 3.6. Suppose that $A$ has toric reduction, so $B=0$ and $E=\mathbf{T}$. The trivialization $t$ amounts to a bilinear pairing $M^{\prime} \times M \rightarrow K^{\times}$such that $-\log |t|$ is nondegenerate. This defines an embedding $M^{\prime} \hookrightarrow \mathrm{T}^{\prime}=\operatorname{Spec}\left(K\left[M^{\prime}\right]\right)$ by the rule $\chi^{u^{\prime}}(u)=t\left(u^{\prime}, u\right)$, and the quotient $\mathrm{T}^{\prime \mathrm{an}} / M^{\prime}$ is canonically isomorphic to $A^{\prime \text { an }}$. See [FvdP04, Chapter 6].

3.7. Line bundles. By descent theory, one can study line bundles on $A$ (equivalently, on $A^{\text {an }}$ ) by studying line bundles on $E^{\text {an }}$ with an $M^{\prime}$-linearization. It turns out that it is enough to consider only line bundles on $E^{\text {an }}$ which are pulled back from $B$.

Example 3.8. Recall from (3.2.1) that we can regard $E_{u}$ as an extension of $B$ by $\mathbf{G}_{m}$. The composite homomorphism

$$
\varepsilon_{u}: M^{\prime} \longrightarrow E(K) \stackrel{e_{u}}{\longrightarrow} E_{u}(K)
$$

defines an $M^{\prime}$-linearization of $E_{u}$. This pulls back to an $M^{\prime}$-linearization $\varepsilon_{u}$ of $q^{*} E_{u}$. By [BL91, Corollary 4.10], a cubical line bundle on $E^{\text {an }}$ with $M^{\prime}$-linearization descends to the trivial rigidified line bundle on $A$ if and only if it is isomorphic to $\left(q^{*} E_{u}, \varepsilon_{u}\right)$.

Notation 3.9. For a line bundle $L$ on a group scheme $G$ we set

$$
\mathscr{D}_{2} L=m^{*} L \otimes p_{1}^{*} L^{-1} \otimes p_{2}^{*} L^{-1},
$$

where $p_{1}, p_{2}: G \times G \rightarrow G$ are the two projections and $m: G \times G \rightarrow G$ is multiplication.

Let $G=B$ be an abelian variety and let $L$ be a line bundle on $B$. Recall from (1.1) that $L$ gives rise to a symmetric homomorphism $\varphi_{L}: B \rightarrow B^{\prime}$ defined on points by $x \mapsto T_{x}^{*} L \otimes L^{-1}$. This is equivalent to the "universal" identity $\left(\operatorname{Id} \times \varphi_{L}\right)^{*} P=\mathscr{D}_{2} L$. 
The following theorem is the non-Archimedean analogue of Riemann's period relations.

Theorem 3.10 ([BL91, Theorems 6.7 and 6.13]). There is a canonical bijective correspondence between the following data:

(1) Rigidified line bundles on A.

(2) Equivalence classes of triples $(L, \lambda, c)$, where $L$ is a rigidified line bundle on $B$, $\lambda: M^{\prime} \rightarrow M$ is a group homomorphism, and $c: M^{\prime} \rightarrow \Phi^{*} L$ is a trivialization (as a rigidified line bundle on $M^{\prime}$ ), satisfying

$$
\Phi^{\prime} \circ \lambda=\varphi_{L} \circ \Phi \quad \text { and } \quad c\left(u_{1}^{\prime}+u_{2}^{\prime}\right) \otimes c\left(u_{1}^{\prime}\right)^{-1} \otimes c\left(u_{2}^{\prime}\right)^{-1}=t\left(u_{1}^{\prime}, \lambda\left(u_{2}^{\prime}\right)\right)
$$

for all $u_{1}^{\prime}, u_{2}^{\prime} \in M^{\prime}$, where we use the canonical identification $\mathscr{D}_{2} L=\left(\operatorname{Id} \times \varphi_{L}\right)^{*} P$. Two triples $\left(L_{1}, \lambda_{1}, c_{1}\right)$ and $\left(L_{2}, \lambda_{2}, c_{2}\right)$ are equivalent provided that $\lambda_{1}=\lambda_{2}$ and there exists $u \in M$ such that $L_{1} \otimes L_{2}^{-1} \cong E_{u}$ and $c_{1} \otimes c_{2}^{-1} \cong \varepsilon_{u}$, where $\varepsilon_{u}$ is defined in (3.8.1).

The above correspondence is compatible with tensor product in the obvious way. Moreover, the line bundle $L_{A}$ on $A$ corresponding to $(L, \lambda, c)$ is ample if and only if $L$ is ample and the symmetric form $\left(u_{1}^{\prime}, u_{2}^{\prime}\right) \mapsto\left[u_{1}^{\prime}, \lambda\left(u_{2}^{\prime}\right)\right]$ is positive-definite, in which case

$$
\operatorname{dim} H^{0}\left(A, L_{A}\right)=\left[M: \lambda\left(M^{\prime}\right)\right] \operatorname{dim} H^{0}(B, L) .
$$

The correspondence is constructed by descent with respect to the subgroup $M^{\prime} \subset E^{\text {an }}$. The $M^{\prime}$-linearization on $q^{*} L$ defined by a triple in (2) is described as follows, in terms of sections [BL91, Proposition 4.9]: the isomorphism $q^{*} L \stackrel{\sim}{\longrightarrow} T_{u^{\prime}}^{*} q^{*} L$ corresponding to the action of $u^{\prime} \in M^{\prime}$ on $q^{*} L$ is given by $f \mapsto c\left(u^{\prime}\right) \otimes e_{\lambda\left(u^{\prime}\right)} \otimes f$, up to canonical isomorphism. (The canonical isomorphism in question is the pullback via $q$ of $T_{\Phi\left(u^{\prime}\right)}^{*} \cong \cong$ $L\left(\Phi\left(u^{\prime}\right)\right) \otimes E_{\lambda\left(u^{\prime}\right)} \otimes L$, where $L\left(\Phi\left(u^{\prime}\right)\right)$ is the constant line bundle on the fiber of $L$ over $\Phi\left(u^{\prime}\right)$. See $[$ BL91, §4].)

Example 3.11. Suppose that $A$ has toric reduction, as in Example 3.6. Since there are no nontrivial line bundles on $\mathbf{T}^{\text {an }}$, by descent theory, the Picard group $\operatorname{Pic}(A)$ is naturally isomorphic to $H^{1}\left(M^{\prime}, \mathscr{O}\left(\mathrm{T}^{\mathrm{an}}\right)^{\times}\right)$, the group cohomology of $M^{\prime}$ with coefficients in $\mathscr{O}\left(\mathrm{T}^{\mathrm{an}}\right)^{\times}$. Any analytic invertible function on $\mathrm{T}^{\mathrm{an}}$ is in fact algebraic [FvdP04, Theorem 6.3.3(1)], hence is a constant times a character; in other words, $\mathscr{O}\left(\mathrm{T}^{\mathrm{an}}\right)^{\times}=K^{\times} \times M$. Let $Z: u^{\prime} \mapsto$ $Z_{u^{\prime}}$ be a 1-cocycle representing a line bundle $L \in \operatorname{Pic}(A)$. Up to coboundary, we can write $Z_{u^{\prime}}=c\left(u^{\prime}\right) \chi^{\lambda\left(u^{\prime}\right)}$ for some $c\left(u^{\prime}\right) \in K^{\times}$and $\lambda\left(u^{\prime}\right) \in M$, such that $u^{\prime} \mapsto \lambda\left(u^{\prime}\right)$ is a homomorphism from $M^{\prime}$ to $M$, and for $u_{1}^{\prime}, u_{2}^{\prime} \in M^{\prime}$ one has

$$
c\left(u_{1}^{\prime}+u_{2}^{\prime}\right) c\left(u_{1}^{\prime}\right)^{-1} c\left(u_{2}^{\prime}\right)^{-1}=\chi^{\lambda\left(u_{2}^{\prime}\right)}\left(u_{1}^{\prime}\right)=t\left(u_{1}^{\prime}, \lambda\left(u_{2}^{\prime}\right)\right)
$$

and $c(0)=1$. A triple corresponding to the line bundle $L$ via Theorem 3.10 is $(\mathscr{O}, \lambda, c)$. For $u \in M$, the coboundary of $\chi^{u} \in \mathscr{O}\left(\mathrm{T}^{\mathrm{an}}\right)^{\times}$is $u^{\prime} \mapsto t\left(u^{\prime}, u\right)$, so that $(\mathscr{O}, \lambda, c)$ and $(\mathscr{O}, \lambda, t(\cdot, u) c)$ define the same line bundle. Unwrapping the definitions and using Example 3.6, we find that the trivialization $\varepsilon_{u}$ of (3.8.1) reduces to the homomorphism $t(\cdot, u)$ in this case. 
3.12. Translations of line bundles. To $x^{\prime} \in E^{\prime}(K)$ we can associate the translationinvariant line bundle $L_{x^{\prime}}$ on $B$ corresponding to $q^{\prime}\left(x^{\prime}\right) \in B^{\prime}(K)$ : that is, $L_{x^{\prime}}=P_{B \times q^{\prime}\left(x^{\prime}\right)}$, where $P \rightarrow B \times B^{\prime}$ is the Poincaré bundle. For $u^{\prime} \in M^{\prime}$ we have $E_{u^{\prime}}^{\prime}=P_{\Phi\left(u^{\prime}\right) \times B^{\prime}}$, so $e_{u^{\prime}}\left(x^{\prime}\right) \in$ $P_{\Phi\left(u^{\prime}\right) \times q^{\prime}\left(x^{\prime}\right)}=\left(L_{x^{\prime}}\right)_{\Phi\left(u^{\prime}\right)}$. It turns out that $u^{\prime} \mapsto e_{u^{\prime}}\left(x^{\prime}\right)$ defines a homomorphism $c_{x^{\prime}}: M^{\prime} \rightarrow$ $L_{x^{\prime}}$ over $\Phi: M^{\prime} \rightarrow B$, where we regard $L_{x^{\prime}}$ as an extension of $B$ by $\mathbf{G}_{m}$. In fact more is true:

Proposition 3.13 ([BL91, Corollaries 4.11 and 4.12]). The association $x^{\prime} \mapsto\left(L_{x^{\prime}}, c_{x^{\prime}}\right)$ defines a bijection between points of $E^{\prime}(K)$ and isomorphism classes of pairs $(L, c)$, where $L$ is a translation-invariant line bundle on $B$ and $c: M^{\prime} \rightarrow L$ is a homomorphism lifting $\Phi: M^{\prime} \rightarrow$ B. Moreover, $\left(L_{x^{\prime}}, 0, c_{x^{\prime}}\right)$ is a triple as in Theorem 3.10 giving rise to the translation-invariant line bundle on A corresponding to $p^{\prime}\left(x^{\prime}\right) \in A^{\prime}(K)$.

See also the paragraph before the statement of Theorem 6.8 in [BL91]. When $x^{\prime}=$ $u \in M \subset E^{\prime}(K)$ we have $q^{\prime}(u)=\Phi^{\prime}(u)$, so $L_{u}=E_{u}$. Since $e_{u^{\prime}}(u)=e_{u}\left(u^{\prime}\right)$ it follows that $c_{u}=\varepsilon_{u}$ as in Example 3.8, so $\left(L_{u}, 0, c_{u}\right)$ gives rise to the trivial line bundle on $A$. This is consistent with Theorem 3.10 and the fact that $p^{\prime}(u)=0 \in A^{\prime}(K)$.

Proposition 3.14. Let $L_{A}$ be a line bundle on $A$, and let $(L, \lambda, c)$ be a triple corresponding to $L_{A}$ as in Theorem 3.10. Let $\varphi=\varphi_{L_{A}}: A \rightarrow A^{\prime}$ be the homomorphism induced by $L_{A}$ and let $\widetilde{\varphi}: E^{\mathrm{an}} \rightarrow E^{\mathrm{an}}$ be the lift to universal covers. Let $x \in E(K), y=p(x) \in A(K)$, $z=q(x) \in B(K)$, and $x^{\prime}=\tilde{\varphi}(x) \in E^{\prime}(K)$. Then $T_{z}^{*} L \cong L_{x^{\prime}} \otimes L$, and $\left(T_{z}^{*} L, \lambda, c_{x^{\prime}} \otimes c\right)$ is a triple corresponding to $T_{y}^{*} L_{A}$.

Proof. As explained in (3.23) below, we have $q^{\prime} \circ \tilde{\varphi}=\varphi_{L} \circ q$. By definition $L_{x^{\prime}}$ is the line bundle defined by $q^{\prime}\left(x^{\prime}\right)=q^{\prime}(\tilde{\varphi}(x))$, and $T_{z}^{*} L \otimes L^{-1}$ is the line bundle defined by $\varphi_{L}(z)=\varphi_{L}(q(x))$. Hence $L_{x^{\prime}} \cong T_{z}^{*} L \otimes L^{-1}$. Similarly, $T_{y}^{*} L_{A} \otimes L_{A}^{-1}$ is the line bundle defined by $\varphi(y) \in A^{\prime}(K)$; as $\varphi(y)=p^{\prime}(\tilde{\varphi}(x))=p^{\prime}\left(x^{\prime}\right)$, this line bundle corresponds to the triple $\left(L_{x^{\prime}}, 0, c_{x^{\prime}}\right)$ by Proposition 3.13. The result follows by compatibility of the formation of these triples with tensor product.

Definition 3.15. With the notation in Proposition 3.14, we call the triple $\left(T_{z}^{*} L, \lambda, c_{x^{\prime}} \otimes c\right)$ the translate of $(L, \lambda, c)$ by $x \in E(K)$, and we write

$$
T_{x}^{*}(L, \lambda, c):=\left(T_{z}^{*} L, \lambda, c_{x^{\prime}} \otimes c\right) .
$$

Example 3.16. Suppose that $A$ has toric reduction, as in Examples 3.6 and 3.11. A point $x^{\prime} \in \mathbf{T}^{\prime}(K)$ is equivalent to a homomorphism $c_{x^{\prime}}: M^{\prime} \rightarrow K^{\times}$, and the cocycle $Z: u^{\prime} \mapsto$ $c_{x^{\prime}}\left(u^{\prime}\right)$ represents a translation-invariant line bundle on $A$, as explained in Example 3.11. This cocycle is a coboundary if and only if $x^{\prime}=u \in M$, in which case $c_{u}\left(u^{\prime}\right)=\chi^{u^{\prime}}(u)=$ $\chi^{u}\left(u^{\prime}\right)=t\left(u^{\prime}, u\right)$.

If $L_{A}$ is a line bundle on $A$ with corresponding cocycle $Z_{u^{\prime}}=c\left(u^{\prime}\right) \chi^{\lambda\left(u^{\prime}\right)}$, then $T_{y}^{*} L_{A}$ corresponds to the cocycle $T_{x^{\prime}}^{*} Z$. One calculates $\left(T_{x^{\prime}}^{*} Z\right)_{u^{\prime}}=c_{x^{\prime}}\left(u^{\prime}\right) c\left(u^{\prime}\right) \chi^{\lambda\left(u^{\prime}\right)}$, which we write as the triple $\left(\mathscr{O}, \lambda, c_{x^{\prime}} \cdot c\right)$.

3.17. Theta functions. We will also need an analytic description of sections of line bundles on $A$, building on Theorem 3.10. At this point it is convenient to pass to invertible sheaves, following [BL91, §4]. Our correspondence between invertible sheaves and line 
bundles is contravariant: if $L$ is a line bundle then its corresponding invertible sheaf is $\mathscr{L}=\mathscr{H} \mathrm{om}(L, \mathscr{O})$. In particular, a section $s$ of $L$ gives rise to a section of $\mathscr{L}^{-1}$, also denoted $s$.

Let $\mathscr{L}$ be a rigidified invertible sheaf on $B$. There is a canonical decomposition of $H^{0}\left(E^{\mathrm{an}}, q^{*} \mathscr{L}\right)$ as a certain completed direct sum

$$
H^{0}\left(E^{\mathrm{an}}, q^{*} \mathscr{L}\right)=\bigoplus_{u \in M}^{\wedge} H^{0}\left(B, \mathscr{L} \otimes \mathscr{E}_{u}\right) \otimes e_{u},
$$

where $\mathscr{E}_{u}$ is the invertible sheaf on $B$ corresponding to $E_{u}$ and $e_{u} \in H^{0}\left(B, \mathscr{E}_{u}^{-1}\right)$. In other words, any $f \in H^{0}\left(E^{\text {an }}, q^{*} \mathscr{L}\right)$ has a canonical Fourier decomposition

$$
f=\sum_{u \in M} a_{u} \otimes e_{u} \quad a_{u} \in H^{0}\left(B, \mathscr{L} \otimes \mathscr{E}_{u}\right)
$$

Remark 3.18. Let $V \subset B^{\text {an }}$ be a formal affinoid over which the formal Raynaud extension (3.3.1) splits, and which trivializes $\mathscr{L}$ (as a formal line bundle). Then $q^{-1}(V) \cong$ $V \times \mathrm{T}$, and $\left.f\right|_{V}$ has a unique Laurent series decomposition $f=\sum_{u \in M} a_{u} \chi^{u}$ with $a_{u} \in \mathscr{O}(V)$. The Fourier decomposition (3.17.1) is the globalization of this decomposition. Moreover, for $x \in E^{\text {an }}$ one has

$$
\|f(x)\|_{q^{*} \mathscr{L}}=\left|\sum a_{u} \chi^{u}(x)\right|
$$

essentially by the definition of the model metric $\|\cdot\|_{\mathscr{L}}$. (This also explains the convergence in the completed direct sum above.) See [BL91, §5].

Proposition 3.19 ([BL91, Proposition 5.2]). Let $\mathscr{L}_{A}$ be a rigidified invertible sheaf on $A$, and let $(\mathscr{L}, \lambda, c)$ be a corresponding triple in Theorem 3.10. Let $f \in H^{0}\left(E^{\text {an }}, q^{*} \mathscr{L}\right)$ have Fourier decomposition $f=\sum_{u \in M} a_{u} \otimes e_{u}$. Then the following are equivalent:

(1) $f$ descends to a global section of $\mathscr{L}_{A}$;

(2) $f=c\left(u^{\prime}\right) \otimes e_{\lambda\left(u^{\prime}\right)} \otimes T_{u^{\prime}}^{*} f$ for all $u^{\prime} \in M^{\prime}$;

(3) for all $\left(u^{\prime}, u\right) \in M^{\prime} \times M$ we have

$$
a_{u+\lambda\left(u^{\prime}\right)}=t\left(u^{\prime}, u\right) \otimes c\left(u^{\prime}\right) \otimes T_{\Phi\left(u^{\prime}\right)}^{*} a_{u},
$$

ignoring canonical isomorphisms.

See the proof of [BL91, Proposition 5.2] and the internal references therein to unwrap the canonical isomorphisms involved. For our purposes they are not important; we will only use Corollary 3.21, which is a consequence.

Definition 3.20. A global section $f \in H^{0}\left(E^{\mathrm{an}}, q^{*} \mathscr{L}\right)$ which descends to a section of $\mathscr{L}_{A}$ is called a theta function with respect to $(\mathscr{L}, \lambda, c)$. We say that $f$ is invariant under the linearization action of $M^{\prime}$.

We will usually call $f$ simply a "theta function for $\mathscr{L}$ ". Note that theta functions for $\mathscr{L}$ are not intrinsic to $\mathscr{L}_{A}$, as there are multiple triples $(\mathscr{L}, \lambda, c)$ giving rise to $\mathscr{L}_{A}$.

Corollary 3.21. With the notation in Proposition 3.19, for $x \in B^{\text {an }}$ and $\left(u^{\prime}, u\right) \in M^{\prime} \times M$ we have

$$
\left\|a_{u+\lambda\left(u^{\prime}\right)}(x)\right\|_{\mathscr{L} \otimes \mathscr{E}_{u}}=\left\|t\left(u^{\prime}, u\right)\right\|_{P} \cdot\left\|c\left(u^{\prime}\right)\right\|_{\mathscr{L}} \cdot\left\|a_{u}\left(x+\Phi\left(u^{\prime}\right)\right)\right\|_{\mathscr{L} \otimes \mathscr{E}_{u}}
$$

if $f$ is a theta function. 
Proof. It follows from Proposition 3.19 that

$$
a_{u+\lambda\left(u^{\prime}\right)}=t\left(u^{\prime}, u\right) \otimes c\left(u^{\prime}\right) \otimes T_{\Phi\left(u^{\prime}\right)}^{*} a_{u},
$$

where we have used the canonical isomorphisms

$$
T_{u^{\prime}}^{*} q^{*} \mathscr{L}=q^{*} T_{\Phi\left(u^{\prime}\right)}^{*} \mathscr{L}=q^{*} \mathscr{L}\left(\Phi\left(u^{\prime}\right)\right) \otimes q^{*} \mathscr{E}_{u} \otimes q^{*} \mathscr{L}, \quad T_{u^{\prime}}^{*} \mathscr{E}_{u}=\mathscr{E}_{u}
$$

By the construction of the formal metric, it follows that the sections of these line bundles will differ, via this isomorphisms, by an element of norm 1 . This shows the compatibility of the metrics which proves the claim.

Example 3.22. Suppose that $A$ has toric reduction, as in Examples 3.6, 3.11, and 3.16. Let $L$ be a line bundle on $A$, corresponding to a cocycle $u^{\prime} \mapsto c\left(u^{\prime}\right) \chi^{\lambda\left(u^{\prime}\right)}$ as in Example 3.11. An analytic function on $\mathbf{T}$ has the form (Fourier decomposition) $f=\sum_{u \in M} a_{u} \chi^{u}$ for $a_{u} \in$ $K$, where $\operatorname{val}\left(a_{u}\right)+\langle u, v\rangle \rightarrow \infty$ on the complements of finite subsets of $M$ for all $v \in N_{\mathrm{R}}$. The function $f$ descends to a section of $\mathscr{L}$ if and only if $f=c\left(u^{\prime}\right) \chi^{\lambda\left(u^{\prime}\right)} T_{u^{\prime}}^{*} f$ for all $u^{\prime} \in M^{\prime}$, i.e., if and only if

$$
\sum a_{u+\lambda\left(u^{\prime}\right)} \chi^{u+\lambda\left(u^{\prime}\right)}=c\left(u^{\prime}\right) \chi^{\lambda\left(u^{\prime}\right)} \sum a_{u} \chi^{u}\left(u^{\prime}\right) \chi^{u}=\sum t\left(u^{\prime}, u\right) c\left(u^{\prime}\right) a_{u} \chi^{u+\lambda\left(u^{\prime}\right)} .
$$

This recovers $(3.19 .1)$ in the totally degenerate case.

3.23. Polarizations. Let $L_{A}$ be a rigidified line bundle on $A$, with corresponding triple $(L, \lambda, c)$ as in Theorem 3.10. Let $\varphi_{L_{A}}: A \rightarrow A^{\prime}$ be the homomorphism induced by $L_{A}$ and let $\widetilde{\varphi}_{L_{A}}: E^{\text {an }} \rightarrow E^{\text {an }}$ be the lift to universal covers, so $\widetilde{\varphi}_{L_{A}}\left(M^{\prime}\right) \subset M$. It is automatic that $\tilde{\varphi}_{L_{A}}\left(\mathrm{~T}^{\mathrm{an}}\right) \subset \mathrm{T}^{\mathrm{an}}$, so $\widetilde{\varphi}_{L_{A}}$ induces homomorphisms $\lambda_{\mathrm{T}}: \mathrm{T} \rightarrow \mathrm{T}^{\prime}$ and $\lambda_{B}: B \rightarrow B^{\prime}$. See [BL91, Proposition 3.5].

Proposition 3.24. [BL91, Proposition 6.10, Remark 6.11] In the above situation, the following homomorphisms $M^{\prime} \rightarrow M$ coincide with $\lambda$ :

(1) The homomorphism on character groups induced by $\lambda_{\mathrm{T}}$.

(2) The restriction of $\tilde{\varphi}_{L_{A}}$ to $M^{\prime}$.

Moreover, $\lambda_{B}=\varphi_{L}$.

By Theorem 3.10, $\varphi_{L_{A}}$ is a polarization if and only if $L$ is ample and $[\cdot, \lambda(\cdot)]$ is positivedefinite. In this case the degrees are related by

$$
\operatorname{deg}\left(\varphi_{L_{A}}\right)=\left[M: \lambda\left(M^{\prime}\right)\right]^{2} \operatorname{deg}\left(\varphi_{L}\right)
$$

See also [BL91, Theorem 6.15].

If $\varphi_{L_{A}}$ is a principal polarization then $\lambda$ is an isomorphism and $\varphi_{L}$ is also a principal polarization. In this case there is a unique nonzero global section $a_{0} \in H^{0}(B, L)$ up to scaling. Taking $u=0$ in (3.19.1), the unique theta function $f$ of $L$ (up to scaling) has Fourier expansion

$$
f=\sum_{u^{\prime} \in M^{\prime}} 1 \otimes c\left(u^{\prime}\right) \otimes T_{\Phi\left(u^{\prime}\right)}^{*} a_{0} \otimes e_{\lambda\left(u^{\prime}\right)} .
$$

We call $f$ the Riemann theta function associated to $(L, \lambda, c)$.

Recall that for $y \in A(K)$, the line bundle $T_{y}^{*} L_{A}$ defines the same principal polarization $\varphi_{L_{A}}$ 
Proposition 3.25. With the above notation, suppose that $\varphi_{L_{A}}$ is a principal polarization. Then there exists $y \in A(K)$ and a triple $(L, \lambda, c)$ for $T_{y}^{*} L_{A}$ such that, if $f=\sum_{u \in M} a_{u} \otimes e_{u}$ is the Fourier expansion of the Riemann theta function on $L$, then for $u^{\prime} \in M^{\prime}$ and $x \in B^{\text {an }}$, we have

$$
\left\|a_{\lambda\left(u^{\prime}\right)}(x)\right\|_{L \otimes E_{u}}=\left\|t\left(u^{\prime}, \lambda\left(u^{\prime}\right)\right)\right\|_{P}^{1 / 2} \cdot\left\|a_{0}\left(x+\Phi\left(u^{\prime}\right)\right)\right\|_{L \otimes E_{u}} \cdot
$$

Moreover, we can choose $y$ and $(L, \lambda, c)$ such that

$$
\left((-1)^{*} L, \lambda,(-1)^{*} c\right)=(L, \lambda, c),
$$

where $(-1): B \rightarrow B$ is negation and $(-1)^{*} c\left(u^{\prime}\right)=c\left(-u^{\prime}\right)$.

Proof. By Corollary 3.21, for the first assertion we only need to show that there exist $y$ and $(L, \lambda, c)$ such that

$$
\left\|c\left(u^{\prime}\right)\right\|_{L}=\left\|t\left(u^{\prime}, \lambda\left(u^{\prime}\right)\right)\right\|_{P}^{1 / 2}
$$

for all $u^{\prime} \in M^{\prime}$. Let $\left(L^{\prime}, \lambda, c^{\prime}\right)$ be a triple associated with $L_{A}$. Let $Q^{\prime}\left(u^{\prime}\right)=-\log \left\|c^{\prime}\left(u^{\prime}\right)\right\|_{L^{\prime}}$ and let $\beta\left(u_{1}^{\prime}, u_{2}^{\prime}\right)=\left[u_{1}^{\prime}, \lambda\left(u_{2}^{\prime}\right)\right]=-\log \left\|t\left(u_{1}^{\prime}, \lambda\left(u_{2}^{\prime}\right)\right)\right\|_{P}$. Then $\beta: M^{\prime} \times M^{\prime} \rightarrow \mathbf{R}$ is a symmetric, positive-definite bilinear form by Theorem 3.10 (since $L_{A}$ is ample), and for $u_{1}^{\prime}, u_{2}^{\prime} \in M^{\prime}$ we have $\beta\left(u_{1}^{\prime}, u_{2}^{\prime}\right)=Q^{\prime}\left(u_{1}^{\prime}+u_{2}^{\prime}\right)-Q^{\prime}\left(u_{1}^{\prime}\right)-Q^{\prime}\left(u_{2}^{\prime}\right)$ (also by Theorem 3.10). Letting $Q(u)=\frac{1}{2} \beta(u, u)$ be the quadratic form associated to the bilinear form $\beta$, we also have $\beta\left(u_{1}^{\prime}, u_{2}^{\prime}\right)=Q\left(u_{1}^{\prime}+u_{2}^{\prime}\right)-Q\left(u_{1}^{\prime}\right)-Q\left(u_{2}^{\prime}\right)$, so $Q-Q^{\prime}: M^{\prime} \rightarrow \mathbf{R}$ is a homomorphism. Since $c\left(u^{\prime}\right)$ and $t\left(u^{\prime}, \lambda\left(u^{\prime}\right)\right)$ are $K$-valued points, the image of $Q-Q^{\prime}$ is contained in $\Gamma:=\operatorname{val}\left(K^{\times}\right)$. Therefore we may regard $Q-Q^{\prime}$ as a point in $N_{\Gamma}^{\prime}:=\operatorname{Hom}\left(M^{\prime}, \Gamma\right) \subset N_{\mathrm{R}}^{\prime}$.

Choose $x^{\prime} \in E^{\prime}(K)$ such that $\operatorname{trop}\left(x^{\prime}\right)=Q-Q^{\prime}$. Let $L_{x^{\prime}}$ be the translation-invariant line bundle on $\beta$ and $c_{x^{\prime}}: M^{\prime} \rightarrow L_{x^{\prime}}$ the homomorphism which correspond to $x^{\prime}$ by Proposition 3.13. For $u^{\prime} \in M^{\prime}$ we have $c_{x^{\prime}}\left(u^{\prime}\right)=e_{u^{\prime}}\left(x^{\prime}\right)$, so

$$
\left\langle u^{\prime}, \operatorname{trop}\left(x^{\prime}\right)\right\rangle=-\log \left\|c_{x^{\prime}}\left(u^{\prime}\right)\right\|_{E_{u^{\prime}}}
$$

by definition of trop (3.2.2). On the other hand,

$$
\left\|c_{x^{\prime}}\left(u^{\prime}\right)\right\|_{E_{u^{\prime}}}=\left\|c_{x^{\prime}}\left(u^{\prime}\right)\right\|_{P}=\left\|c_{x^{\prime}}\left(u^{\prime}\right)\right\|_{L_{x^{\prime}}}
$$

since any translation-invariant line bundle is a pullback of the Poincaré bundle as a formal line bundle. It follows that $Q-Q^{\prime}=-\log \left\|c_{x^{\prime}}(\cdot)\right\|_{L_{x^{\prime}}} \in N_{\Gamma}^{\prime}$.

Let $\tilde{\varphi}_{L_{A}}: E^{\text {an }} \stackrel{\sim}{\longrightarrow} E^{\text {an }}$ be the lift of $\varphi_{L_{A}}$ to universal covers, let $x=\widetilde{\varphi}_{L_{A}}^{-1}\left(x^{\prime}\right)$, let $y=$ $p(x) \in A(K)$, and let $z=q(x) \in B(K)$. Then $(L, \lambda, c):=T_{x}^{*}\left(L^{\prime}, \lambda^{\prime}, c^{\prime}\right)=\left(T_{z}^{*} L^{\prime}, \lambda, c_{x^{\prime}} \otimes c^{\prime}\right)$ is a triple for $T_{y}^{*} L_{A}$ by Proposition 3.14, and by construction, $-\log \left\|c\left(u^{\prime}\right)\right\|_{L}=Q\left(u^{\prime}\right)=$ $\frac{1}{2} \beta\left(u^{\prime}, u^{\prime}\right)$.

Now we treat the final assertion. To begin we replace $L_{A}$ by $T_{y}^{*} L_{A}$ and $\left(L^{\prime}, \lambda, c^{\prime}\right)$ by $(L, \lambda, c)$. A triple for the translation-invariant line bundle $(-1)^{*} L_{A} \otimes L_{A}^{-1}$ is

$$
\left((-1)^{*} L \otimes L^{-1}, 0,(-1)^{*} c \otimes c^{-1}\right) .
$$

For $u^{\prime} \in M^{\prime}$ we have $\left\|c\left(u^{\prime}\right)\right\|_{L}=\left\|c\left(-u^{\prime}\right)\right\|_{L}$ by (3.25.1), so $\left\|(-1)^{*} c\left(u^{\prime}\right) \otimes c\left(u^{\prime}\right)^{-1}\right\|_{(-1)^{*} L_{L^{-1}}}=$ 1. Let $x^{\prime} \in E^{\prime}(K)$ be the point giving rise to the pair $\left((-1)^{*} L \otimes L^{-1},(-1)^{*} c \otimes c^{-1}\right)$ in the manner of Proposition 3.13, and choose $x^{\prime \prime} \in E^{\prime}(K)$ such that $2 x^{\prime \prime}=x^{\prime}$. Then 
$L_{x^{\prime \prime}}^{\otimes 2} \cong(-1)^{*} L \otimes L^{-1}$ and $c_{x^{\prime \prime}}^{\otimes 2} \cong(-1)^{*} c \otimes c^{-1}$. Since $(-1)^{*} L_{x^{\prime \prime}}=L_{x^{\prime \prime}}^{-1}$ as $L_{x^{\prime \prime}}$ is translationinvariant, we have

$$
(-1)^{*}\left(L \otimes L_{x^{\prime \prime}}\right) \cong(-1)^{*} L \otimes L_{x^{\prime \prime}}^{-1} \cong L \otimes L_{x^{\prime \prime}},
$$

and similarly for $c \otimes c_{x^{\prime \prime}}$. Hence the triple

$$
\left(L \otimes L_{x^{\prime \prime}}, \lambda, c \otimes c_{x^{\prime \prime}}\right)=T_{x^{\prime \prime}}^{*}(L, \lambda, c)
$$

is invariant under the symmetry $(-1)$, and for $u^{\prime} \in M^{\prime}$,

$$
\left\|c_{x^{\prime \prime}}(u)\right\|_{L_{x^{\prime \prime}}}=\sqrt{\left\|(-1)^{*} c\left(u^{\prime}\right) \otimes c\left(u^{\prime}\right)^{-1}\right\|_{(-1) * L \otimes L^{-1}}}=1 .
$$

It follows that $T_{x^{\prime \prime}}^{*}(L, \lambda, c)$ satisfies all of the hypotheses of the Proposition.

Example 3.26. Suppose that $A$ has toric reduction, as in Examples 3.6, 3.11, 3.16, and 3.22. Let $L_{A}$ be a line bundle on $A$, with associated cocycle $u^{\prime} \mapsto c\left(u^{\prime}\right) \chi^{\lambda\left(u^{\prime}\right)}$. Let $\lambda_{\mathrm{T}}: \mathrm{T} \rightarrow \mathrm{T}^{\prime}$ be the homomorphism inducing $\lambda: M^{\prime} \rightarrow M$ on character groups. By (3.11.1) the bilinear form $\left(u_{1}^{\prime}, u_{2}^{\prime}\right) \mapsto t\left(u_{1}^{\prime}, \lambda\left(u_{2}^{\prime}\right)\right)=\chi^{\lambda\left(u_{2}^{\prime}\right)}\left(u_{1}^{\prime}\right)$ is symmetric. Hence for $u_{1}^{\prime}, u_{2}^{\prime} \in$ $M^{\prime} \times M^{\prime}$, we have

$$
\chi^{u_{2}^{\prime}}\left(\lambda_{\mathrm{T}}\left(u_{1}^{\prime}\right)\right)=\chi^{\lambda\left(u_{2}^{\prime}\right)}\left(u_{1}^{\prime}\right)=t\left(u_{1}^{\prime}, \lambda\left(u_{2}^{\prime}\right)\right)=t\left(u_{2}^{\prime}, \lambda\left(u_{1}^{\prime}\right)\right)=\chi^{u_{2}^{\prime}}\left(\lambda\left(u_{1}^{\prime}\right)\right)
$$

where the first equality is the definition of $\lambda_{\mathrm{T}}$ and the last is the definition of the embed$\operatorname{ding} M \hookrightarrow \mathrm{T}^{\prime}$ in Example 3.6. Hence $\lambda$ is the restriction of $\lambda_{\mathrm{T}}$ to $M^{\prime}$, as in Proposition 3.24.

Suppose that $\varphi_{L_{A}}$ is a principal polarization. Choose a bilinear form $\sqrt{t(\cdot, \lambda(\cdot))}: M^{\prime} \times$ $M^{\prime} \rightarrow K^{\times}$whose square is $t(\cdot, \lambda(\cdot)$ ) (choose square roots of the images of pairs of basis elements). Then

$$
f=\sum_{u^{\prime} \in M^{\prime}} \sqrt{t\left(u^{\prime}, \lambda\left(u^{\prime}\right)\right)} \chi^{\lambda\left(u^{\prime}\right)}
$$

is the Riemann theta function associated to the triple $(\mathscr{O}, \lambda, c)$ for $c\left(u^{\prime}\right)=\sqrt{t\left(u^{\prime}, \lambda\left(u^{\prime}\right)\right)}$. See also [Tei88, Definitions 16,19].

\section{TROPICALIZATION OF THETA FUNCTIONS}

An abelian variety $A$ over $K$ has a canonical skeleton $\Sigma$, which is a tropical abelian variety in the sense of Definition 2.6. In this section we define the tropicalization $f_{\text {trop }}$ of a theta function $f$ on $A$, and we prove that $f_{\text {trop }}$ is a tropical theta function on $\Sigma$. We show that a Riemann theta function tropicalizes to the tropical Riemann theta function in the principally polarized case, up to translation and scaling.

Throughout this section we use the notation (3.4) for the uniformization of $A$ and its dual.

4.1. The skeleton. There is a canonical continuous section $\sigma: N_{\mathbf{R}} \rightarrow E^{\text {an }}$ of the tropicalization map trop: $E^{\text {an }} \rightarrow N_{\mathbf{R}}$ defined in (3.2.2), which is constructed in [Gub10, Example 7.2] as follows. Choose a local section $V \rightarrow E^{\text {an }}$ of the formal Raynaud extension (3.3.1) as in Remark 3.3, so $q^{-1}(V) \cong \mathbf{T} \times V$. Then $\operatorname{trop}^{-1}(v) \cong U_{v} \times V$, where $U_{v}$ 
is the inverse image of $v$ under trop: $\mathrm{T}^{\mathrm{an}} \rightarrow N_{\mathrm{R}}$. Any analytic function $h$ on $U_{v} \times V$ has a unique Laurent series expansion

$$
h=\sum_{u \in M} a_{u} \chi^{u} \quad \text { such that } \quad\left|a_{u}\right|_{\text {sup }} \exp (-\langle u, v\rangle) \longrightarrow 0
$$

on the complements of finite sets of $M$, where $a_{u} \in \mathscr{O}(V)$ and $|\cdot|_{\text {sup }}$ is the supremum norm on $\mathscr{O}(V)$. We define $\sigma(v)$ to be the norm on $\mathscr{O}\left(U_{v} \times V\right)$ given by

$$
|h(\sigma(v))|=\max _{u \in M}\left\{\left|a_{u}\right|_{\text {sup }} \exp (-\langle u, v\rangle)\right\} .
$$

This is independent of the choice of $V$ and the choice of splitting (of the formal Raynaud extension), and the resulting section $\sigma: N_{\mathrm{R}} \rightarrow E^{\text {an }}$ is continuous.

Lemma 4.2. Let $\mathfrak{B}$ be the smooth abelian $R$-scheme with generic fiber $B$. There is a unique point $\xi \in B^{\text {an }}$ whose reduction is the generic point of $\mathfrak{B}_{s}$, and $q(\sigma(v))=\xi$ for all $v \in N_{\mathbf{R}}$.

Proof. The first assertion is a standard application of [Ber90, Proposition 2.4.4(ii)]: the point $\xi$ corresponds to the supremum norm on $\mathscr{O}(V)$ for any $V$ as in (4.1). If $h=a_{0}$ is the pullback of a function on $V$ then by definition $|h(\sigma(v))|=\left|a_{0}\right|_{\text {sup }}$, so $q(\sigma(v))=\xi$.

Lemma 4.3. Let $v \in N_{\mathrm{R}}$ and $x \in E(K)$. Then $\sigma(v)+x:=T_{x}(\sigma(v))$ is equal to $\sigma(v+$ $\operatorname{trop}(x))$.

Proof. Let $w=\operatorname{trop}(x)$, choose $V$ as in (4.1), and let $h$ be an analytic function on $\mathrm{T}^{\text {an }} \times V$. We can write $h=\sum_{u \in M} a_{u} \chi^{u}$ for $a_{u} \in \mathscr{O}(V)$, such that $\left|a_{u}\right|_{\text {sup }} \exp \left(-\left\langle v^{\prime}, u\right\rangle\right) \rightarrow 0$ for all $v^{\prime} \in N_{\mathrm{R}}$. We have

$$
\begin{aligned}
\left|h\left(T_{x}(\sigma(v))\right)\right| & =\left|\sum a_{u} T_{x}^{*}\left(\chi^{u}\right)(\sigma(v))\right| \\
& =\max \left\{\left|a_{u}\right|_{\text {sup }} \exp (-\langle u, v\rangle-\langle u, w\rangle)\right\} \\
& =|h(\sigma(v+w))|
\end{aligned}
$$

because $T_{x}^{*}\left(\chi^{u}\right)=\chi^{u}(x) \chi^{u}$ and $\left|\chi^{u}(x)\right|=\exp (-\langle u, \operatorname{trop}(x)\rangle)$ by definition of trop.

Definition 4.4. The skeleton of $A$ is the real torus $N_{\mathrm{R}} / \operatorname{trop}\left(M^{\prime}\right)$.

Note that the pairing $[\cdot, \cdot]: M^{\prime} \times M \rightarrow \mathbf{R}$ of (3.4.2) defines the embedding trop: $M^{\prime} \rightarrow$ $N_{\mathrm{R}}$ used in Definition 4.4. The tropicalization map trop: $E^{\mathrm{an}} \rightarrow N_{\mathrm{R}}$ defines by passage to the quotient a proper, surjective homomorphism $\tau: A^{\text {an }} \rightarrow \Sigma$, and the section $\sigma$ restricts fiberwise to a section $\sigma: \Sigma \rightarrow A^{\text {an }}$. The composition $\sigma \circ \tau: A^{\text {an }} \rightarrow A^{\text {an }}$ is the image of a deformation retraction onto $\sigma(\Sigma)$ by [Ber90, §6.5].

Example 4.5. Suppose that $A$ has toric reduction, as in Examples 3.6, 3.11, 3.16, 3.22, and 3.26. Then $E^{\text {an }}=\mathbf{T}^{\text {an }}$, and for $v \in N_{\mathbf{R}}$ the point $\sigma(v)$ is defined by

$$
|f(\sigma(v))|=\sup _{u \in M}\left|a_{u}\right| \exp (-\langle u, v\rangle) \quad \text { for } \quad f=\sum_{u \in M} a_{u} \chi^{u} .
$$

In this case $a_{u} \in K$ are constants. The map $\sigma: N_{\mathrm{R}} \rightarrow \mathrm{T}^{\text {an }}$ is the usual skeleton of a torus. 
4.6. Skeletons and polarizations. Suppose now that $A$ is endowed with a polarization $\varphi: A \rightarrow A^{\prime}$ associated to an ample line bundle $L_{A}$. By (3.23), $L_{A}$ gives rise to a triple $(L, \lambda, c)$ such that the real-valued pairing

$$
[\cdot, \lambda(\cdot)]: M^{\prime} \times M^{\prime} \longrightarrow \mathbf{R}
$$

is bilinear, symmetric, and positive-definite. The dual abelian variety $A^{\prime}$ has skeleton $\Sigma^{\prime}:=N_{\mathrm{R}}^{\prime} / \operatorname{trop}(M)$, where $N_{\mathrm{R}}^{\prime}=\operatorname{Hom}\left(M^{\prime}, \mathbf{R}\right)$ and trop: $M \rightarrow N_{\mathrm{R}}^{\prime}$ is again defined by $[\cdot, \cdot]$. From now on we identify $M^{\prime}$ and $M$ with their images in $N_{\mathrm{R}}$ and $N_{\mathrm{R}}^{\prime}$, respectively.

Proposition 4.7. The pairing $[\cdot, \cdot]: M^{\prime} \times M \rightarrow \mathbf{R}$ and the homomorphism $\lambda: M^{\prime} \rightarrow M$ make $\Sigma=N_{\mathrm{R}} / M^{\prime}$ into a tropical abelian variety in the sense of Definition 2.6, with dual $\Sigma^{\prime}=N_{\mathbf{R}}^{\prime} / M$. Moreover, the square

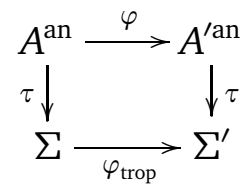

is commutative, where $\varphi_{\text {trop }}$ is the polarization defined by $\lambda$.

Proof. The homomorphism $\lambda: M^{\prime} \rightarrow M$ gives rise to a map $\widetilde{\varphi}_{\text {trop }}: N_{\mathrm{R}} \rightarrow N_{\mathrm{R}}^{\prime}$ making the square

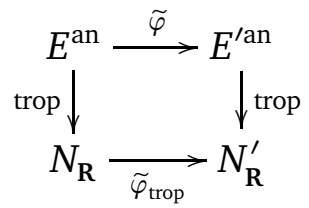

commutative. Since $\tilde{\varphi}$ restricts to $\lambda: M^{\prime} \rightarrow M$ on sublattices by Proposition 3.24, $\widetilde{\varphi}_{\text {trop }}$ also restricts to $\lambda$ on $M^{\prime} \subset N_{\mathrm{R}}$, and hence descends to a homomorphism $\varphi_{\text {trop }}: \Sigma \rightarrow \Sigma^{\prime}$. Unwinding the definitions, for $u_{1}^{\prime}, u_{2}^{\prime} \in M^{\prime}$ we have

$$
\left\langle u_{1}^{\prime}, \lambda\left(u_{2}^{\prime}\right)\right\rangle=-\log \left\|t\left(u_{1}^{\prime}, \lambda\left(u_{2}^{\prime}\right)\right)\right\|_{P}=\left[u_{1}^{\prime}, \lambda\left(u_{2}^{\prime}\right)\right]
$$

(the first being the evaluation pairing $M^{\prime} \times N_{\mathbf{R}}^{\prime} \rightarrow \mathbf{R}$ ), which is analogous to (3.26.1).

See also [BR15, (3.7) and §4] for a discussion in the principally polarized case.

4.8. Tropicalization of theta functions. Fix a rigidified line bundle $L_{A}$ on $A$, let $(L, \lambda, c)$ be a corresponding triple of Theorem 3.10, and let $\varphi_{L_{A}}: A \rightarrow A^{\prime}$ be the induced homomorphism. Let $f \in H^{0}\left(E^{\text {an }}, q^{*} L\right)$ be a nonzero theta function (Definition 3.20).

Definition 4.9. The tropicalization of $f$ is the function

$$
f_{\text {trop }}: N_{\mathbf{R}} \rightarrow \mathbf{R} \quad \text { defined by } \quad f_{\text {trop }}(v)=-\log \|f(\sigma(v))\|_{q^{*} L} \cdot
$$

Here $\|\cdot\|_{q^{*} L}$ is the pullback of the canonical model metric $\|\cdot\|_{L}$ of (3.1). The next proposition shows that $\|f(\sigma(v))\|_{q^{*} L} \neq 0$ for all $v \in N_{\mathbf{R}}$.

Theorem 4.10 (The tropicalization of a theta function is a tropical theta function). Let $f \in H^{0}\left(E^{\mathrm{an}}, q^{*} L\right)$ be a nonzero theta function with tropicalization $f_{\text {trop }}: N_{\mathbf{R}} \rightarrow \mathbf{R}$ and Fourier expansion $f=\sum_{u \in M} a_{u} \otimes e_{u}$. Let $\xi \in B^{\text {an }}$ be the point defined in Lemma 4.2. 
(1) $f_{\text {trop }}(v)=\min _{u \in M}\left\{-\log \left\|a_{u}(\xi)\right\|_{L \otimes E_{u}}+\langle u, v\rangle\right\}<\infty$ for all $v \in N_{\mathbf{R}}$.

(2) $f_{\text {trop }}$ is a piecewise integral affine function.

(3) Suppose that $L_{A}$ is ample, so that $\varphi_{L_{A}}$ is a polarization. Let $c_{\text {trop }}\left(u^{\prime}\right)=-\log \left\|c\left(u^{\prime}\right)\right\|_{L^{\prime}}$. Then

$c_{\text {trop }}\left(u_{1}^{\prime}+u_{2}^{\prime}\right)-c_{\text {trop }}\left(u_{1}^{\prime}\right)-c_{\text {trop }}\left(u_{2}^{\prime}\right)=\left[u_{1}^{\prime}, \lambda\left(u_{2}^{\prime}\right)\right]$ for all $u_{1}^{\prime}, u_{2}^{\prime} \in M^{\prime}$,

and for $u^{\prime} \in M^{\prime}$ and $v \in N_{\mathrm{R}}$ we have

$$
f_{\text {trop }}(v)=f_{\text {trop }}\left(v+u^{\prime}\right)+c_{\text {trop }}\left(u^{\prime}\right)+\left\langle\lambda\left(u^{\prime}\right), v\right\rangle .
$$

In particular, $f_{\text {trop }}$ is a tropical theta function in the sense of Definition 2.9.

Proof. Choose a local splitting $q^{-1}(V) \cong \mathbf{T} \times V$ of the formal Raynaud extension (3.3.1) which also trivializes $L$, as in Remark 3.3. Note that $\Sigma \subset V$ by Lemma 4.2. On $q^{-1}(V)$ we can write

$$
f=\sum_{u \in M} a_{u} \chi^{u} \quad \text { where } \quad a_{u} \in \mathscr{O}(V),
$$

and some $a_{u}$ is nonzero. We have

$$
\|f\|_{q^{*} L}=\left|\sum a_{u} \chi^{u}(\sigma(v))\right|=\max _{u \in M}\left\{\left|a_{u}\right|_{\text {sup }} \exp (-\langle u, v\rangle)\right\}=\max _{u \in M}\left\{\left|a_{u}(\xi)\right| \exp (-\langle u, v\rangle)\right\} .
$$

by (3.18.1), (4.1.1), and Lemma 4.2. This proves (1). Clearly $f$ is piecewise affine, and the slopes are integral because the linear part of $f$ on any domain of linearity has the form $\langle u, \cdot\rangle$, which takes integer values on $N$ (note $\left|a_{u}(\xi)\right|$ is constant with respect to $v$ ); this proves (2).

The identity for $c_{\text {trop }}(\cdot)$ is obtained by applying logarithm to the identity for $c(\cdot)$ in Theorem 3.10. By Proposition 3.19 we have $f=c\left(u^{\prime}\right) \otimes e_{\lambda\left(u^{\prime}\right)} \otimes T_{u}^{*} f$ for all $u^{\prime} \in M^{\prime}$. Therefore

$$
\|f(\sigma(v))\|_{q^{*} L}=\left\|c\left(u^{\prime}\right)\right\|_{L} \cdot\left\|e_{\lambda\left(u^{\prime}\right)}(\sigma(v))\right\|_{E_{\lambda\left(u^{\prime}\right.} \cdot} \cdot\left\|f\left(\sigma(v)+u^{\prime}\right)\right\|_{q^{*} L} \cdot
$$

We have

$$
-\log \left\|e_{\lambda\left(u^{\prime}\right)}(\sigma(v))\right\|_{E_{\lambda\left(u^{\prime}\right)}}=\left\langle\lambda\left(u^{\prime}\right), \operatorname{trop}(\sigma(v))\right\rangle=\left\langle\lambda\left(u^{\prime}\right), v\right\rangle
$$

by definition of trop (3.2.2). Since $\sigma(v)+u^{\prime}=\sigma\left(v+u^{\prime}\right)$ by Lemma 4.3, this completes the proof.

4.11. Theta functions and translation. Before discussing the Riemann theta function, we mention how tropicalization of theta functions behaves with respect to translation. This is somewhat complicated by the fact that theta functions are only defined in the presence of a triple $(L, \lambda, c)$, which must also be translated. Let $L_{A}$ be a rigidified line bundle on $A$. By Theorem 3.10, it has an associated triple $(L, \lambda, c)$, where $L$ is a rigidified line bundle on $B$. Recall that we have an algebraic morphism $q: E \rightarrow B$ and an analytic morphism $p: E^{\text {an }} \rightarrow A^{\text {an }}$ appearing in the exact sequences (1) and (2), respectively. Fix a nonzero theta function $f \in H^{0}\left(E^{\text {an }}, q^{*} L\right)$ and a point $x \in E(K)$. We wish to explain how translation of $f$ by $x$ interacts with tropicalization. To this end, denote the images of $x$ under $p$ and $q$ by $y=p(x) \in A(K)$ and $z=q(x) \in B(K)$, respectively. Let $\varphi_{L_{A}}: A \rightarrow A^{\prime}$ be the homomorphism induced by $L_{A}$, as described in 1.1, and let $\widetilde{\varphi}_{L_{A}}: E \rightarrow E^{\prime}$ be its lift to the universal covers $E$ of $A$ and $E^{\prime}$ of $A^{\prime}$. Define $x^{\prime} \in E^{\prime}(K)$ to be the image of $x$ under 
$\varphi_{L_{A}}$. By Proposition 3.14, $\left(T_{z}^{*} L, \lambda, c_{x^{\prime}} \otimes c\right)$ is a triple for $T_{y}^{*} L_{A}$. It is clear that we have canonical isomorphisms of line bundles

$$
T_{x}^{*} q^{*} L \cong q^{*} T_{z}^{*} L \quad \text { on } E
$$

and

$$
T_{x}^{*} p^{*} L_{A} \cong p^{*} T_{y}^{*} L_{A} \quad \text { on } \quad E^{\text {an }} \text {. }
$$

Since $f$ is a theta function, there exists a unique section $f_{A} \in H^{0}\left(A, L_{A}\right)$ such that $f=p^{*} f_{A}$. Thus $T_{x}^{*} f=p^{*} T_{y}^{*} f_{A} \in H^{0}\left(E^{\text {an }}, q^{*} T_{z}^{*} L\right)$ is a theta function with respect to the translated triple $\left(T_{z}^{*} L, \lambda, c_{x^{\prime}} \otimes c\right)=T_{x}^{*}(L, \lambda, c)$.

Lemma 4.12. With the above notation, $\left(T_{x}^{*} f\right)_{\text {trop }}=T_{\text {trop }(x)}^{*} f_{\text {trop }}$ for all $x \in E(K)$, where for any $v \in N_{\mathrm{R}}$, we define

$$
\left(T_{v}^{*} f_{\text {trop }}\right)(w):=f_{\text {trop }}(v+w) .
$$

Proof. The canonical metric on $T_{z}^{*} L$ coincides with the pullback via $T_{z}$ of $\|\cdot\|_{L}$ since translation by $z$ extends to the special fiber of the smooth model of $B$. The Lemma follows from this and Lemma 4.3.

Theorem 4.13 (The tropicalization of a Riemann theta function is a tropical Riemann theta function). Let $L_{A}$ be an ample rigidified line bundle on $A$ defining a principal polarization. Let $(L, \lambda, c)$ be a triple corresponding to $L_{A}$ and let $f \in H^{0}\left(E^{\text {an }}, q^{*} L\right)$ be the Riemann theta function for $L$, as defined in (3.24.1). Then there exists $x \in E(K)$ such that $\left(T_{x}^{*} f\right)_{\text {trop }}=T_{\text {trop }(x)}^{*} f_{\text {trop }}$ is equal to the tropical Riemann theta function associated to $\lambda$, up to an additive constant.

Proof. By Proposition 3.25, Theorem 4.10(1), and Lemma 4.2, there is a translate of $(L, \lambda, c)$ whose Riemann theta function tropicalizes to the tropical Riemann theta function (up to an additive constant). The translate of $f$ must be the Riemann theta function of this translate, so the Corollary follows from Lemma 4.12.

\section{APPLICATION TO CONSTRUCTING RATIONAL FUNCTIONS}

We apply the results of $\S 4$ to give a method for tropically constructing rational functions $f$ on $A$ such that $-\log |f|$ has a prescribed behavior on the skeleton $\Sigma$. First we remark that if $f$ is a nonzero rational function on $A$, then $|f(x)| \neq 0$ for all $x \in \Sigma$ : this follows from Theorem 4.10 as applied to the trivial line bundle.

Definition 5.1. Let $f$ be a nonzero rational function on $A$. Its tropicalization is the function

$$
f_{\text {trop }}: \Sigma \longrightarrow \mathbf{R} \text { defined by } f_{\text {trop }}(v)=-\log |f(\sigma(v))| \text {. }
$$

The composition $f \circ p: E^{\text {an }} \rightarrow \mathbf{R}$ is a theta function with respect to the trivial line bundle, and the composition of $f_{\text {trop }}$ with the quotient map $N_{\mathbf{R}} \rightarrow \Sigma$ coincides with ( $f \circ$ $p)_{\text {trop }}$ (Definition 4.9). It follows from Theorem 4.10 that $f_{\text {trop }}$ is piecewise integral affine (Definition 2.3). In what follows we will identify functions on $\Sigma$ with functions on $N_{\mathbf{R}}$ which are invariant under the translation action of $M^{\prime}$. 
Theorem 5.2. For $i=1,2$ let $L_{i, A}$ be an ample line bundle on $A$, let $\left(L_{i}, \lambda_{i}, c_{i}\right)$ be a triple corresponding to $L_{i, A}$ via Theorem 3.10, and let $f_{i} \in H^{0}\left(E^{\text {an }}, q^{*} L_{i}\right)$ be a nonzero theta function with tropicalization $f_{i, \text { trop. }}$. Suppose that $f_{1, \text { trop }}-f_{2, \text { trop }}$ is invariant under translation by elements of $M^{\prime}$, i.e., that $f_{1, \text { trop }}$ and $f_{2 \text {,trop }}$ satisfy the same transformation law (4.10.1). Then there exists a rational function $h$ on $A$ such that $h_{\text {trop }}=f_{1, \text { trop }}-f_{2, \text { trop }}$.

Proof. Let $f=f_{1} / f_{2}$, regarded as a section of $q^{*}\left(L_{1} \otimes L_{2}^{-1}\right)$. A triple corresponding to $L:=L_{1, A} \otimes L_{2, A}^{-1}$ is $(L, \lambda, c):=\left(L_{1} \otimes L_{2}^{-1}, \lambda_{1}-\lambda_{2}, c_{1} \otimes c_{2}^{-1}\right)$. Clearly $\lambda_{i}$ is determined by (4.10.1) (fix $u^{\prime}$ and vary $v$ ), so $\lambda_{1}=\lambda_{2}$ and $\lambda=0$. The Fourier expansion for $f$ has the form $f=\sum_{u \in M} a_{u} \otimes e_{u}$, and satisfies the invariance property (3.19.1), which in this case says

$$
a_{u}=t\left(u^{\prime}, u\right) \otimes c\left(u^{\prime}\right) \otimes T_{\Phi\left(u^{\prime}\right)}^{*} a_{u}
$$

for all $\left(u^{\prime}, u\right) \in M^{\prime} \times M$. Define $f^{\prime}=a_{0} \otimes e_{0} \in H^{0}\left(E^{\text {an }}, q^{*} L\right)$. Then $f^{\prime}$ is also a theta function, and $f_{\text {trop }}^{\prime}$ is constant by Theorem 4.10(1). Multiplying $f^{\prime}$ by a scalar, we may assume $f_{\text {trop }}^{\prime}$ is identically zero. Then $h=f / f^{\prime}$ is a rational function on $A$ such that $h_{\text {trop }}=f_{\text {trop }}$.

5.3. Example constructions. Here we give concrete examples of Theorem 5.2. These are translations of standard constructions into this tropical/non-Archimedean setting.

Lemma 5.4. Let $L_{A}$ be an ample rigidified line bundle on $A$ with associated triple $(L, \lambda, c)$. Choose points $x_{1}, \ldots, x_{n} \in E(K)$ such that $\sum_{i=1}^{n} x_{i}=0$. Then $\bigotimes_{i=1}^{n} T_{x_{i}}^{*}(L, \lambda, c)$ is canonically isomorphic to $\left(L^{\otimes n}, n \lambda, c^{\otimes n}\right)$.

Proof. Let $\varphi=\varphi_{L_{A}}: A \rightarrow A^{\prime}$, let $\tilde{\varphi}: E^{\text {an }} \rightarrow E^{\text {an }}$ be the lift to universal covers, and let $y_{i}=p\left(x_{i}\right) \in A(K)$ and $x_{i}^{\prime}=\widetilde{\varphi}\left(x_{i}\right) \in E^{\prime}(K)$. Then $T_{x_{i}}^{*}(L, \lambda, c)=\left(L_{x_{i}^{\prime}} \otimes L, \lambda, c_{x_{i}^{\prime}} \otimes c\right)$, where $L_{x_{i}^{\prime}}$ and $c_{x_{i}^{\prime}}$ are defined in (3.12). We have $\bigotimes_{i=1}^{n} L_{x_{i}^{\prime}}=L_{\sum x_{i}^{\prime}}=\mathscr{O}$ since $L_{x_{i}^{\prime}}$ is the translation-invariant line bundle on $B$ corresponding to $q^{\prime}\left(x_{i}^{\prime}\right) \in B^{\prime}(K)$. Moreover, for fixed $u^{\prime} \in M^{\prime}$ we have $c_{x_{i}^{\prime}}\left(u^{\prime}\right)=e_{u^{\prime}}\left(x_{i}^{\prime}\right) \in P_{\Phi\left(u^{\prime}\right) \times B^{\prime}}=E_{u^{\prime}}^{\prime}$; since $e_{u^{\prime}}$ is a homomorphism, $\sum_{i=1}^{n} c_{x_{i}^{\prime}}\left(u^{\prime}\right)=0$.

Definition 5.5. Fix an ample line bundle $L_{A}$ giving rise to a principal polarization $\varphi_{L_{A}}: A \rightarrow$ $A^{\prime}$, and fix a triple $(L, \lambda, c)$ corresponding to $L_{A}$. A level-n theta function with respect to $(L, \lambda, c)$ is a theta function associated to $\left(L^{\otimes n}, n \lambda, c^{\otimes n}\right)$.

Proposition 5.6. Fix an ample line bundle $L_{A}$ giving rise to a principal polarization $\varphi_{L_{A}}: A \rightarrow$ $A^{\prime}$, and fix a triple $(L, \lambda, c)$ corresponding to $L_{A}$. Let $f \in H^{0}\left(E^{\text {an }}, q^{*} L\right)$ be the Riemann theta function for $L$. Choose points $x_{1}, \ldots, x_{n} \in E(K)$ such that $\sum_{i=1}^{n} x_{i}=0$. Then $\bigotimes_{i=1}^{n} T_{x_{i}}^{*} f$ is a level-n theta function. In particular, $f^{\otimes n}$ is a level-n theta function.

This is immediate from Lemma 5.4 and the discussion in (4.11). It follows that $h=$ $\bigotimes_{i=1}^{n} T_{x_{i}}^{*} f / f^{\otimes n}$ is invariant under $M^{\prime}$ and is therefore a rational function on $A$. By Lemma 4.12, for all $v \in N_{\mathbf{R}}$,

$$
h_{\text {trop }}(v)=\sum_{i=1}^{n} f_{\text {trop }}\left(v+v_{i}\right)-n f_{\text {trop }}(v)
$$


where $v_{i}=\operatorname{trop}\left(x_{i}\right)$. In particular, there exists a rational function on $A$ whose tropicalization is the right side of (5.6.1), where $f_{\text {trop }}$ is the tropical Riemann theta function on $\Sigma$.

5.7. Kummer functions. Certain level-2 theta functions are symmetric with respect to negation. Thus (5.6.1) will often produce tropically Kummer functions, in the sense of Definition 2.4. We wish to prove the other direction: given a tropically Kummer function, we want to construct a rational map which is symmetric with respect to negation.

Let $A /(-1)$ be the quotient in which we identify each pair of points $x$ and $-x$ in $A$, and define $\Sigma /(-1)$ similarly. The former quotient has the structure of an algebraic variety, called the Kummer variety of $A$. The quotienting morphism $A \rightarrow A /(-1)$ is called the Kummer map. See [BL04, §4.8].

Let $A^{\prime}=A /(-1)$. The Kummer map induces an injection $\Sigma /(-1) \hookrightarrow A^{\prime a n}$, and since the deformation retraction $A^{\text {an }} \rightarrow \Sigma$ is canonical, it induces a deformation retraction $A^{\prime \text { an }} \rightarrow \Sigma /(-1)$. We call $\Sigma /(-1)$ the skeleton of the Kummer variety $A^{\prime}$.

Definition 5.8. Let $A$ be an abelian variety over $K$ with skeleton $\Sigma$, and let $f: A \rightarrow \mathbf{G}_{m}^{r}$ be a rational map. We say that $f$ is Kummer if it factors through the Kummer map $A \rightarrow$ $A /(-1)$.

Definition 5.8 applies in particular to nonzero rational functions. If $f$ is a rational map which is Kummer then $f_{\text {trop }}$ is Kummer in the sense of Definition 2.4: that is, it factors through the quotient $\Sigma \rightarrow \Sigma /(-1)$, hence defines a function $f_{\text {trop }}: \Sigma /(-1) \rightarrow \mathbf{R}^{r}$.

Lemma 5.9. Fix an ample line bundle $L_{A}$ giving rise to a principal polarization $\varphi_{L_{A}}: A \rightarrow A^{\prime}$, and fix a triple $(L, \lambda, c)$ corresponding to $L_{A}$. Replacing $L_{A}$ by a translate, we assume that $L_{A}$ and $(L, \lambda, c)$ satisfy the conclusions of Proposition 3.25. Let $f \in H^{0}\left(E^{\mathrm{an}}, q^{*} L\right)$ be the Riemann theta function for $L$, and choose $x \in E(K)$. Then the rational function $h=T_{x}^{*} f \otimes$ $T_{-x}^{*} f \otimes f^{\otimes-2}$ of Proposition 5.6 is Kummer.

Proof. By Proposition 3.25, $(-1)^{*} L=L$ and $(-1)^{*} c=c$, so $(-1)^{*} f$ is also a theta function for $L$. Since $f$ is the only Riemann theta function for $L$ up to scaling, and since $(-1): A \rightarrow$ $A$ fixes the identity, it follows that $(-1)^{*} f=f$. Hence $(-1)^{*} T_{x}^{*} f=T_{-x}^{*}(-1)^{*} f=T_{-x}^{*} f$, so $(-1)^{*} h=h$.

In particular, if $f_{\text {trop }}$ is the tropical Riemann theta function for $\Sigma$, then there exists a rational function $h$ on $A^{\prime}$ such that

$$
h_{\text {trop }}(v)=f_{\text {trop }}(v+w)+f_{\text {trop }}(v-w)-2 f_{\text {trop }}(v),
$$

where $w=\operatorname{trop}(x)$.

\section{REFERENCES}

[Ber90] V. G. Berkovich, Spectral theory and analytic geometry over non-Archimedean fields, Mathematical Surveys and Monographs, vol. 33, American Mathematical Society, Providence, RI, 1990. MR 1070709 (91k:32038)

[BL91] S. Bosch and W. Lütkebohmert, Degenerating abelian varieties, Topology 30 (1991), no. 4, 653698. MR 1133878 (92i:14043)

[BL04] C. Birkenhake and H. Lange, Complex abelian varieties, second ed., Grundlehren der Mathematischen Wissenschaften, vol. 302, Springer-Verlag, Berlin, 2004. MR 2062673 (2005c:14001) 
[BR15] M. Baker and J. Rabinoff, The skeleton of the Jacobian, the Jacobian of the skeleton, and lifting meromorphic functions from tropical to algebraic curves, Int. Math. Res. Not. IMRN (2015), no. 16, 7436-7472.

[FC90] G. Faltings and C.-L. Chai, Degeneration of abelian varieties, Ergebnisse der Mathematik und ihrer Grenzgebiete (3) [Results in Mathematics and Related Areas (3)], vol. 22, Springer-Verlag, Berlin, 1990, With an appendix by David Mumford. MR 1083353 (92d:14036)

[FK17] K. Fujiwara and F. Kato, Foundations of rigid geometry I, 2017, Preprint available at http://www.arxiv.org/abs/1308.4734.

[FvdP04] J. Fresnel and M. van der Put, Rigid analytic geometry and its applications, Progress in Mathematics, vol. 218, Birkhäuser Boston, Inc., Boston, MA, 2004. MR 2014891 (2004i:14023)

[Gub10] W. Gubler, Non-Archimedean canonical measures on abelian varieties, Compos. Math. 146 (2010), no. 3, 683-730. MR 2644932 (2011d:14041)

[Mum72] D. Mumford, An analytic construction of degenerating abelian varieties over complete rings, Compositio Math. 24 (1972), 239-272. MR 0352106 (50 \#4593)

[MZ08] G. Mikhalkin and I. Zharkov, Tropical curves, their Jacobians and theta functions, Curves and abelian varieties, Contemp. Math., vol. 465, Amer. Math. Soc., Providence, RI, 2008, pp. 203230. MR 2457739 (2011c:14163)

[Tei88] J. Teitelbaum, p-adic periods of genus two Mumford-Schottky curves, J. Reine Angew. Math. 385 (1988), 117-151. MR 931217 (89b:14043)

E-mail address: foster@ihes.fr

L'Institut des Hautes Études Scientifiques, Le Bois-Marie, 35 Route de CHARTres, 91440 BuresSUR-YVETTE, FRANCE

E-mail address: rabinof $f @ m a t h . g a t e c h . e d u$

School of Mathematics, Georgia Institute of Technology, Atlanta GA 30332-0160, USA

E-mail address: farbod@math.cornell.edu

Department of Mathematics, Cornell University, IthacA, NeW York 14853-4201, USA

E-mail address: alejandro.soto@uni-tuebingen.de

Eberhard Karls Universität TÜbingen, Fachbereich Mathematik, Auf Der Morgenstelle 10, D72076 TÜBINGEN, GERMANY. 